\title{
Computing the Bond Strength of 3D Printed Polylactic Acid Scaffolds in Mode I and II Using Experimental tests, Finite Element Method and Cohesive Zone Modeling
}

\section{Nogol Nazemzadeh}

Amirkabir University of Technology

Anahita Ahmadi Soufivand ( $\sim$ anahita.a.soufivand@gmail.com )

Amirkabir University of Technology

Nabiollah Abolfathi

Amirkabir University of Technology

\section{Research Article}

Keywords: 3D printing, Finite Element Method, Cohesive Zone Model, Fracture Mechanics, Tissue Engineering

Posted Date: February 13th, 2021

DOI: https://doi.org/10.21203/rs.3.rs-218617/v1

License: (c) (1) This work is licensed under a Creative Commons Attribution 4.0 International License. Read Full License

Version of Record: A version of this preprint was published at The International Journal of Advanced Manufacturing Technology on October 2nd, 2021. See the published version at https://doi.org/10.1007/s00170-021-08124-w. 
Computing the Bond Strength of 3D Printed Polylactic Acid Scaffolds in Mode I and II Using Experimental tests, Finite Element Method and Cohesive Zone Modeling

Nogol Nazemzadeh ${ }^{1}$, Anahita Ahmadi Soufivand ${ }^{1, *}$, Nabiollah Abolfathi ${ }^{1, *}$

${ }^{1}$ Biomedical Engineering Faculty, Amirkabir University of Technology, 424 Hafez Ave, Tehran, Tehran Province 15916, Iran

*Correspondence to:

Nabiollah Abolfathi, Ph.D.

Biomedical Engineering Faculty, Amirkabir University of Technology

424 Hafez Ave, Tehran, Tehran Province 15916, Iran

Phone: +98-21-64542364; Fax: +98-21-66468186; E-mail: nabolfathi@aut.ac.ir

Anahita Ahmadi Soufivand, Ph.D.

Biomedical Engineering Faculty, Amirkabir University of Technology, 424 Hafez Ave, Tehran, Tehran Province 15916, Iran

Phone: +98-912-4491811; E-mail: a.a.soufivand@aut.ac.ir, anahita.a.soufivand@gmail.com 


\section{Abstract}

The advent of the Three-Dimensional (3D) printing technique, as an Additive Manufacturing (AM) technology, made the manufacture of complex porous scaffolds plausible in the tissue engineering field. In Fused Deposition Modeling (FDM) based 3D printing, layer upon layer deposition of filaments produces voids and gaps, leading to a crack generation and loose bonding. Cohesive Zone Model (CZM), a fracture mechanics concept, is a promising theory to study the layers bond behavior. In this paper, a combination of experimental and computational investigations was proposed to obtain bond parameters and evaluate the effect of porosity and microstructure on these parameters. First, we considered two different designs for scaffolds beside a non-porous Bulk design. Then, we performed Double Cantilever Beam (DCB) and Singe Lap Shear (SLS) tests on the 3D printed samples for Modes I and II, respectively. Afterward, we developed the numerical simulations of these tests using the Finite Element Method (FEM) to obtain CZM bond parameters. Results demonstrate that the initial stiffness and cohesive strength were pretty similar for all designs in Mode I. However, the cohesive energy for the Bulk sample was approximately four times of porous samples. Furthermore, for Mode II, the initial stiffness and cohesive energy of the Bulk model were five and four times of porous designs while their cohesive strengths were almost the same. Also, using cohesive parameters was significantly enhanced the accuracy of FEM predictions in comparison with fully bonded assumption. It can be concluded that for the numerical analysis of 3D printed parts mechanical behavior, it is necessary to obtain and suppose the cohesive parameters. The present work illustrates the effectiveness of CZM and FEM combination to obtain the layer adhesive parameters of the 3D printed scaffold. 
Keywords: 3D printing, Finite Element Method, Cohesive Zone Model, Fracture Mechanics, Tissue Engineering

\section{Introduction}

The cutting-edge 3D printing technology fabricates objects by deposition of materials in the layer upon layer fashion [1, 2]. According to ASTM F42, printing processes can be divide into seven subgroups of extrusion-based, powder bed fusion, binder jetting, material jetting, directed energy deposition, sheet lamination, and vat photo-polymerization processes [3]. Extrusion-based FDM, as a straightforward and cost-effective method [4, 5], provides users to fabricate complex threedimensional parts quickly by deposition of the melted filaments through a nozzle on a building platform.

Nowadays, 3D printing technologies have been used in tissue engineering purposes since they allow us to create scaffolds with a complex 3D microstructure and a controllable porosity, pore shape, and size $[6,7]$. The porosity facilitates cells to attach and proliferate by providing easy access to microenvironment supplied nutrition and oxygen [8]. Scaffolds located in the body of a patient must simulate both mechanical and biological properties of the native tissue to bear the mechanical stress of the surrounding microenvironment and not to stimulate the immune system [9].

Various types of biocompatible and biodegradable polymers were used in manufacturing 3D

printed scaffold [10], e.g., Polycarbonate (PC), Polyglycolic acid (PGA), Acrylonitrile Butadiene Styrene (ABS), Polycaprolactone (PCL), and Poly-Lactic Acid (PLA) [11]. PLA is the FDA approved 
hydrophobic aliphatic polyester for use in different medical applications [12]. Besides its biological properties, it has satisfying mechanical properties such as thermal stability, low viscosity, high strength, and high-elastic modulus made PLA a well-suited polymer for the FDM technology [13].

The mechanical properties of additively manufactured PLA bone scaffolds have been investigated by several researchers. Tayton et al. provided a platform to compare mechanical shear properties of bone scaffolds made from PLA and PLA-Hydroxyapatite [14]. Naghieh et al. predicted the mechanical properties of porous PLA bone scaffolds fabricated by FDM [6]. This research reported the scaffold compressive elastic modulus by conducting the experimental compression test and FEM. Gremare et al. performed tensile tests on the 3D printed PLA bone scaffold to examine the effect of pore dimension on the mechanical properties [15]. It is noticeable that the numerical FEM approach was used repeatedly in the literature to predict the mechanical response of FDM parts in silico very well $[1,6,16]$. On the other hand, in the experimental approach, fabrication of numerous specimens and performing mechanical tests to find an appropriate mechanical property for FDM parts are costly and time-consuming.

In the FDM process, some voids and gaps generate among bonded struts because of the circular or elliptical shapes of the extruded filament cross-sections, and juxtaposing these struts does not make a uniform part [17]. So, crack propagation is susceptible to occur between adjacent layers of the part [18], which leads to ultimate failure. This phenomenon can be modeled and analyzed by the fracture mechanics-based CZM.

\subsection{Cohesive Zone Model (CZM)}


The cohesive zone is a softening region located ahead of a crack tip. When stress at the tip reaches the cohesive strength, and enough energy is available for a new cracked area creation, an existing crack starts to propagate. Afterward, separation will occur between two adjacent surfaces across an extended crack [19, 20]. Cohesive zone cracks initiation and propagation can be modeled base on crack opening and needed traction relationship using bilinear tractionseparation law (TSL). In TSL law, it assumes that the relationship between traction and separation consists of two linear regions, shown in Figure 1. Three parameters describe these linear regions: initial stiffness, cohesive strength, and fracture energy, defined in the following section.

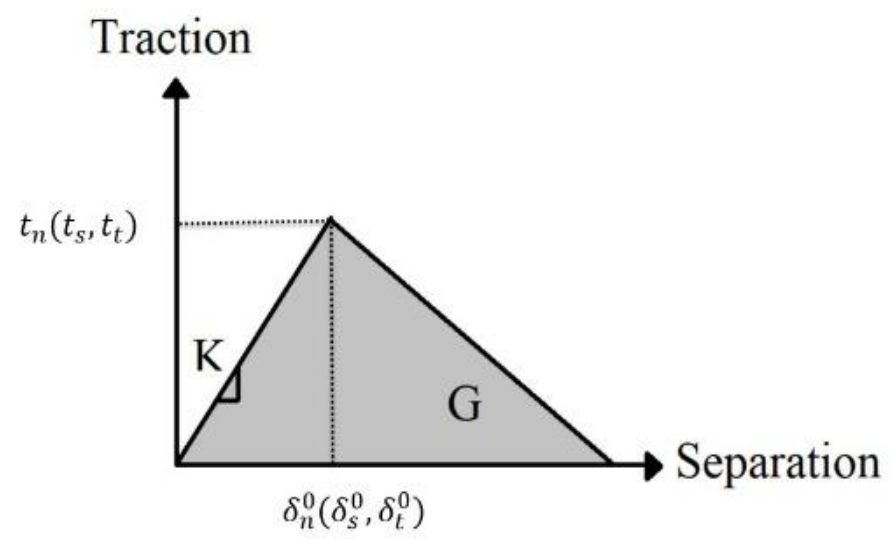

Figure 1. Representation of Cohesive Zone Model (CZM) by linear regions and parameters: initial stiffness $(K)$, cohesive strength $(T)$, and fracture energy $(G)$.

\subsection{Determination of cohesive parameters}

\section{Initial stiffness}

Initial stiffness relates to the initial crack response before reaching cohesive traction to cohesive strength [17]. For calculating the initial stiffness, Equation 1 [21] or setting an artificially high value from $10^{6} \mathrm{~N} / \mathrm{mm}^{3}$ [22] to $10^{8} \mathrm{~N} / \mathrm{mm}^{3}$ [23] can be employed, and in this study, we use the latter method. 


$$
\mathrm{K}=\frac{E}{t}
$$

In Equation $1, E$ is the relevant elastic modulus, and $t$ is the adhesive thickness

\section{Cohesive strength}

Damage initiation determines by the cohesive strength, and after reaching cohesive traction to the cohesive strength, it starts to reduce due to damage accumulation [17]. The adhesive strength calculates from Equation 2.

$$
T=\frac{P_{C}}{A}
$$

Where $P_{c}$ is the critical load, and $A$ is the adhesive area. In this research, we calculated the cohesive strength initially from Equation 2 and then calibrated it.

\section{Cohesive energy}

The fracture energy is equal to the area under a traction-separation curve. The separation will happen when the cohesive zone dissipated energy reaches the fracture energy. The fracture energy release rate of Mode I $\left(G_{l}\right)$ can be obtained from Equation 3, presented in ASTM D5528 [24].

$$
G_{I}=\frac{3 P \delta}{2 b a}
$$

Where $\mathrm{P}$ is the load, $\delta$ is the load point displacement, $\mathrm{b}$ is sample width, and a is debonding length. In this paper, we initially calculated the fracture energy from Equation 3, and then we calibrated it. 
The identification of a cohesive law and its parameters is still highly controversial. There is no effective experimental method to directly measure the parameters like mechanical stresses near the crack tip because the fracture zone is tiny. Therefore, by correlating the experimental data and numerical simulation, inverse techniques such as FEM have been used recently to estimate the CZM parameters [25-30]. Furthermore, fracture mechanics theories have been implemented by researchers to investigate the fractural properties in additively manufactured parts. Kishore et al., by considering the effect of layer temperature on interlayer strength, made an infrared preheating system to increase the overall strength of parts fabricated by additive manufacturing [31]. Spoerk M. et al. extracted optimized printing parameters (layer thickness, layer design, and temperature) to boost inter and intra-layer strength of parts fabricated by FDM [32]. Seppala et al. developed a framework from the polymer interdiffusion viewpoint to understand the interlayer-layer strength in material extruded parts [33]. Recently, researchers have implemented the CZM, a promising theory in fracture mechanics, to describe interlayer delamination, study interlayer fracture, and predict cohesive parameters of AM parts. Liravi et al. developed a framework using experiment and FEM cohesive zone simulation to extract the delamination force of a bottom-up constrained surface projection-based stereolithography (SLA) [34]. Ahmadi et al. implemented CZM in the FEM package to understand the effects of various microstructural properties on the macroscopic mechanical properties of selective laser melting (SLM) parts [35]. Spackman et al. developed a CZM based FEM model to capture the adhesion behavior of fiber-reinforced soft composite additive manufactured parts [36]. Park et al. characterized the fracture behavior of truss structure parts manufactured by the material extrusion process by proposing a modified DCB test and CZM [17]. The modified model consists 
of two adjacent layers, where the CZM is defined between these two layers to achieve interlayer strength. Fonseca et al. proposed an experimental procedure and CZM inverse procedure to characterize interlaminar fracture in Mode I loading of fused filament fabricated (FFF) parts [37]. To this aim, they conducted the DCB experiment for 3D printed parts, which are manufactured by two types of material: pure and short fiber reinforced Polyamide 12 (PA12). This experimental procedure was followed with numerical simulation to extract CZM parameters.

Researchers have investigated the adhesive-bonded joint in additively manufacture parts by conducting single lap shear (SLS) test. S. Kumar et al. evaluated the potential of 3D printed multimaterial adhesive interfaces by exploring their compliance-tailored adhesive designs using the SLS test [38]. Kovan et al. performed the SLS test on PLA printed part to investigate the effect of print orientation and layer thickness on the bonding strength of FDM parts [39]. Garcia et al. developed a framework using the SLS test and FEM for manufacturing an optimum bonded joint design [40]. Falck et al., by a novel AM approach to fabricate hybrid structures of layered metalpolymer, considered the adhesive bonding of parts [41]. Dugbenoo E. et al. present a new technique for increasing the bonding surface to improve bond strength and toughness of parts manufactured by FDM [42].

Previous researches can divide into two main groups: one group focused on the experimental and numerical investigation of scaffold mechanical properties manufactured by FDM. Another group concentrated on CZM modeling of FDM parts and conducting DCB and SLS tests. Our research goal is investigating the bonding strength of 3D printed scaffolds using the CZM approach instead of assuming fully bonded layers. We conducted this research for Modes I and II and considered the effect of porosity and microstructure on the bond behavior. To reach this 
goal, we develop a research framework composed of experimental and numerical processes, includes mechanical tests of PLA filament tension, DCB, and SLS along with FEM simulations, shown in Figure 2.

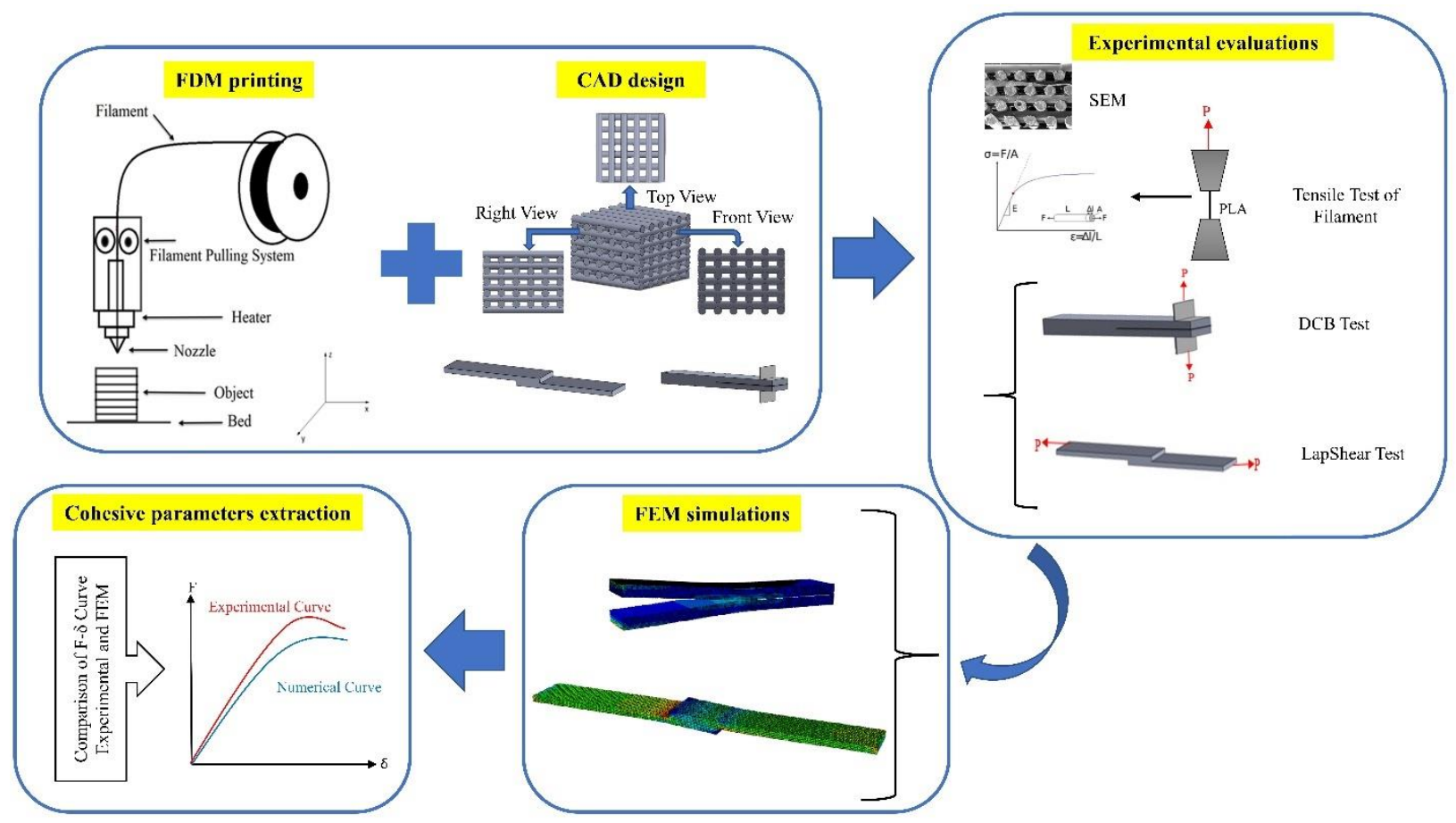

Figure 2. The research framework was exploited in this study. From the top left, initially, we used the FDM parameters to computer-aided design (CAD) of the 3D printing part. After the manufacturing of specimens, we performed the experimental tests on the filament, DCB, and SLS samples. Then, the FEM simulations with various cohesive parameters were applied, and finally, we extracted the CZM parameters for each design and test by comparing numerical and experimental data.

First, the process of designing and manufacturing specimens for mechanical tests will be explained. Then, the procedures for each experimental test will be described. Afterward, the 
experimental method to calculate CZM for Modes I and II and the FEM based numerical modeling containing inverse framework to estimate the cohesive parameters from experimental data will be specified. Then, cohesive parameters of Modes I and II for all designs will be extracted from the curve fitting process. Finally, the mechanical properties of 3D printed parts with and without cohesive assumption in numerical models will be compared.

\section{Material and methods}

\subsection{Specimen fabrication for DCB and SLS tests}

The specimens were fabricated using commercially available white PLA filament with a mean diameter of $1.75 \mathrm{~mm}$, a melting point of $190^{\circ} \mathrm{C}$, and a density of $1.25 \mathrm{~g} \cdot \mathrm{cm}^{-3}$ (ZF Company, China). We performed the printing process using the Quantum 2025 FDM printer (Persia 3DPrinter Co., Iran) equipped with a brass nozzle with an inner diameter of $0.4 \mathrm{~mm}$ and a bed size of $200 * 200$ $\mathrm{mm}^{2}$.

We provide a Bulk design to investigate the porosity effect on the cohesive parameters by comparing the mechanical behavior of porous designs with it. These parts are depicted in Figure 3. Two types of microstructures for scaffold were manufactured and expressed as Lattice and Shifted designs. The difference between these designs was the location of deposited struts in which there was an offset equal to half of the center to center distance of horizontal struts in Shifted design compare to Lattice design. The diameter of each strut is $400 \mu \mathrm{m}$, according to the extrusion nozzle diameter. The layer height was $300 \mu \mathrm{m}$ for all designs to impose enough layer penetration and bonding region. The center to center distances of two adjacent horizontal struts for Bulk and porous designs were 400 and $800 \mu \mathrm{m}$, respectively. It is noticeable that the average pore size of a scaffold for osteons growing is in the range of 300-400 $\mu \mathrm{m}$ [43]. In this study, we 
assume the pore size of the scaffold in this range, $400 \mu \mathrm{m}$, equal to center to center distances of two adjacent horizontal struts.

(a)

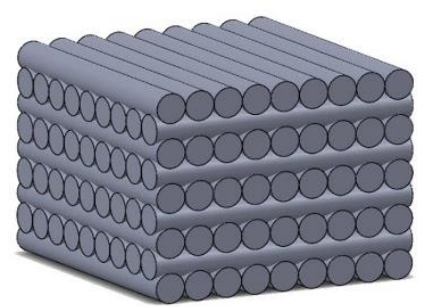

Bulk

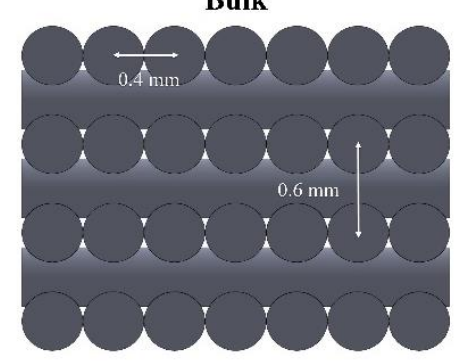

(b)
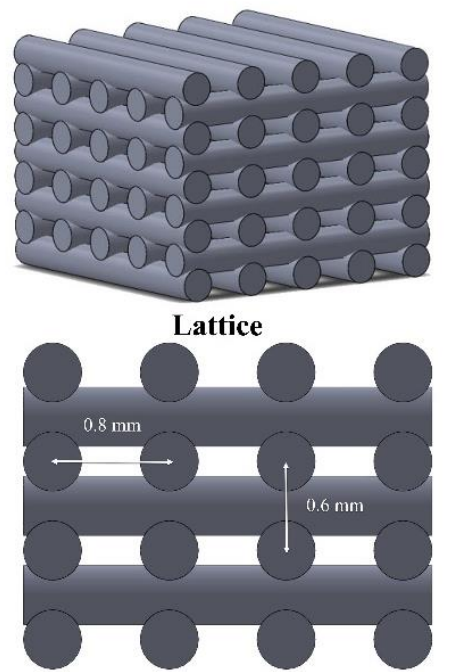

(c)

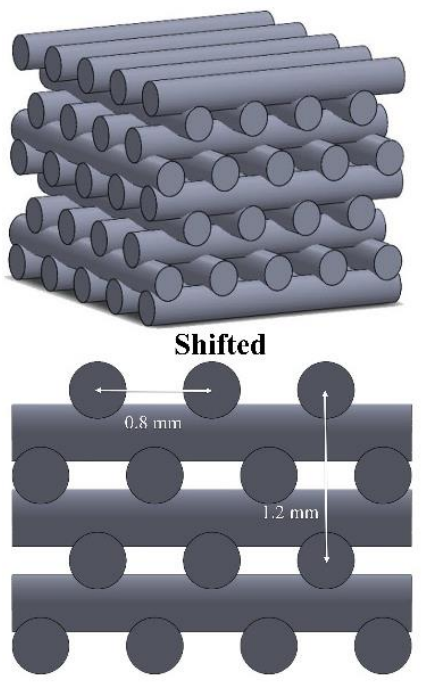

Figure 3. The geometrical specifications of different designs: a) Bulk, b) Lattice, c) Shifted. The FDM parameters were the same for all designs with a strut diameter of $400 \mu \mathrm{m}$ and layer penetration of $100 \mu \mathrm{m}$ as the layer height was $300 \mu \mathrm{m}$.

\subsection{Filament test}

Uniaxial tensile test of extruded filament is challenging because of its small scale and the limitation of fixtures and testing machines. Here, a particular fixture was employed to stabilize the sample during testing (Figure 4). 


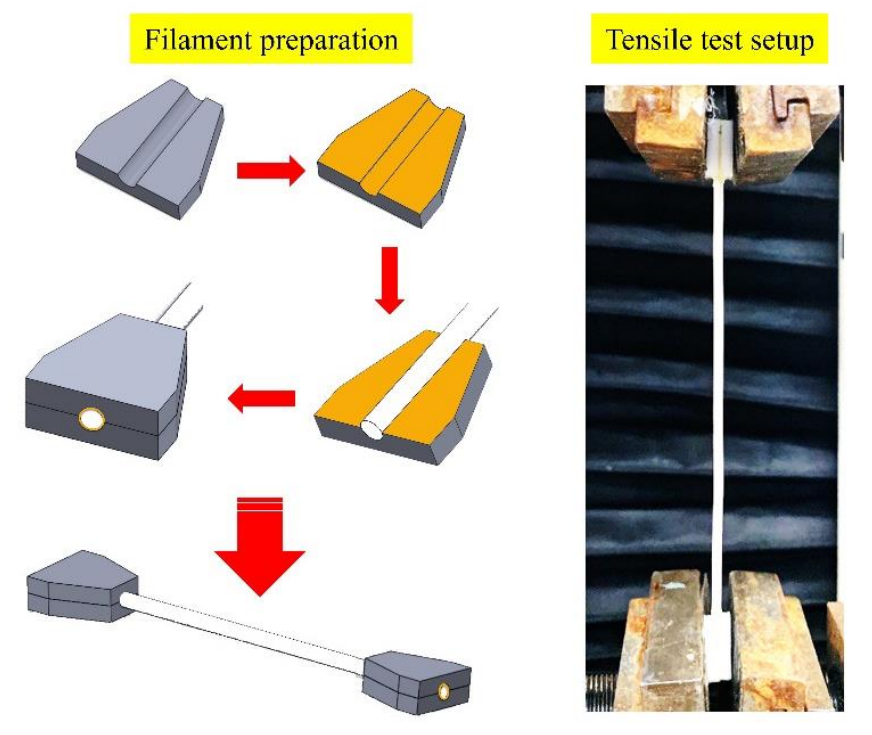

Figure 4. Tensile test configuration for PLA filament. First, the $3 d$ printed PLA dog-bone parts were glued to the filament, and then the tensile test was performed $(n=3)$.

First, trapezoidal PLA tabs similar to the dog-bone specimens head were 3D printed to bind the filaments within the tabs using superglue. We performed the filament test using SANTAM SMT5 (SANTAM ENG. DESIGN CO., Iran) instrumented with a $1 \mathrm{kN}$ load cell at a tension rate of 1 $\mathrm{mm} / \mathrm{min}$. The filaments' lengths were $80 \pm 1 \mathrm{~mm}$, and three filaments were tested. We calculated the elastic modulus from the slope of the initial linear region of the stress-strain curve extracted from the tensile test.

\section{3. $\quad D C B$ test}

The specimens for the DCB test were fabricated according to the ASTM D5528 recommended dimensions, depicted in Figure 5a. A glue tape was placed between the $21^{\text {st }}$ and $22^{\text {nd }}$ layers during the printing process to create an initial crack (Figure 5b) in all designs. Five specimens were manufactured for each design, and the piano hinges were installed to specimens for exerting load 
(Figure 5c). The DCB test was performed using universal testing machine SANTAM SMT-5 (SANTAM ENG. DESIGN CO., Iran) instrumented with a $1 \mathrm{kN}$ load cell (Figure $5 \mathrm{~d}$ ) at an extension rate of $2 \mathrm{~mm} / \mathrm{min}$.

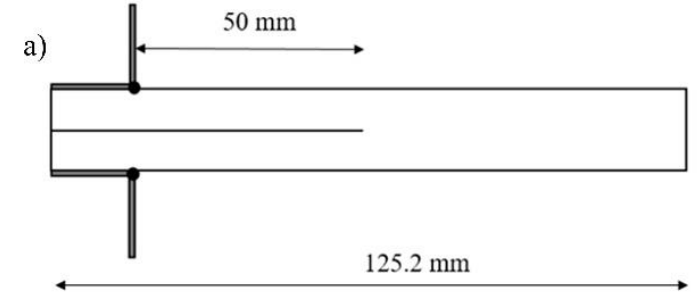

b)

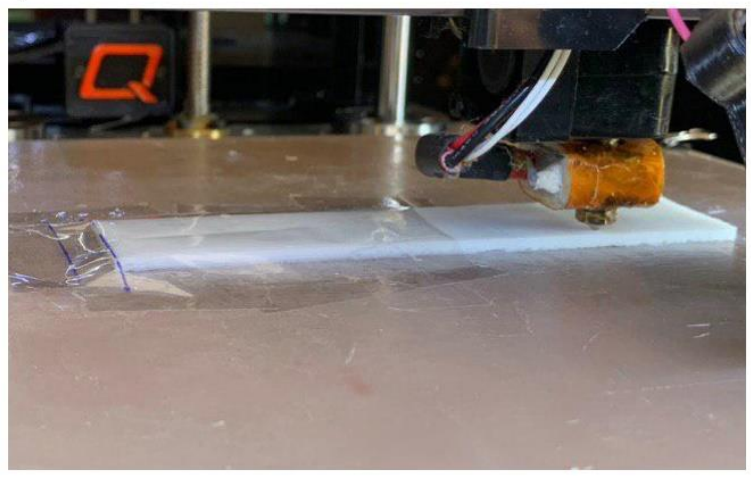

e) Tensile Displacement

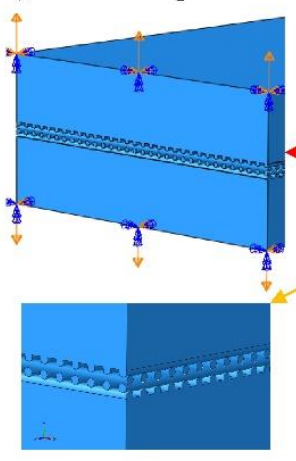

Lattice

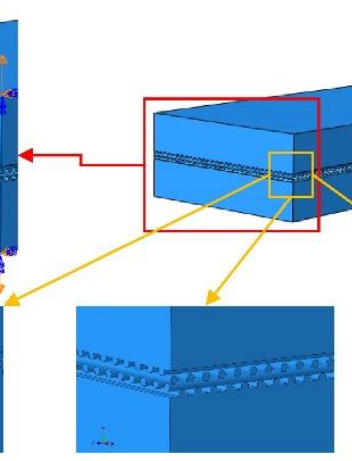

Shifted c)
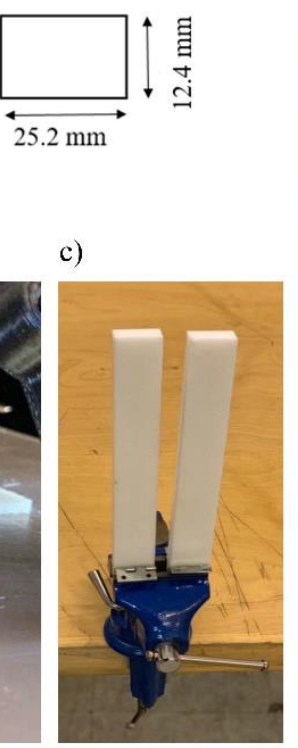

d)

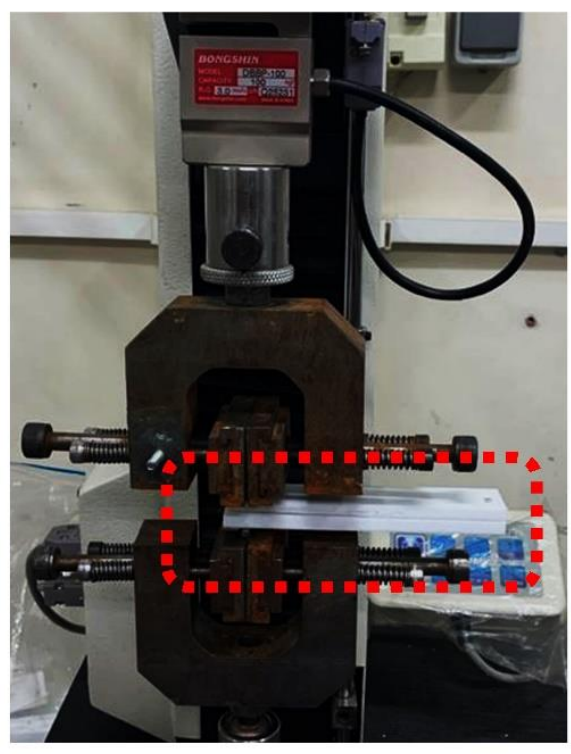

f) ENCASTRE

\section{ENCASTRE}

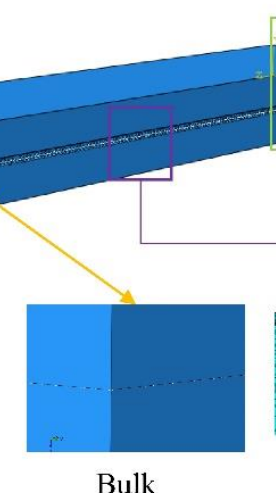

Bulk

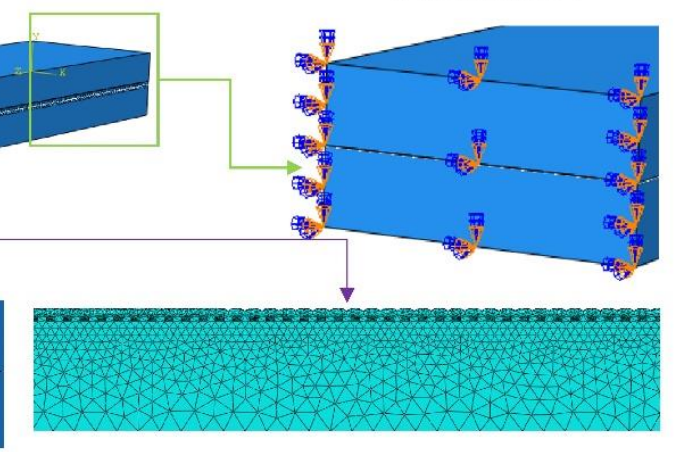

g) 3D Stress Tetrahedral Element

Figure 5. The DCB test specifications in experimental (a-d) and numerical (e-g) studies. (a) The specimen dimensions based on ASTM D5528, (b) Creation of the initial crack during the printing process, (c) Installing piano hinge, (d) Performing DCB test, (e) Tensile displacement to simulate 
loading regime, (f) Constraining all degree of freedom at the end of DCB specimen, (g) Using quadratic tetrahedral (C3D10) elements for meshing of Lattice, Shifted and Bulk designs.

Besides, in attempting to find the cohesive parameters of Mode I by analyzing the DCB fracture test in ABAQUS, a 3D model of DCB has been implemented, shown in Figure 5(e-g). In order to reduce the time of calculation, we modeled four layers of the porous scaffold.

For defining the isotropic elastic material model for PLA, Young's modulus was measured as 3180 MPa from the tensile filament test, explained in section 2.2, and the Poisson's ratio was chosen 0.36 based on the related study [44]. Also, we simulated the loading condition in the test by the tensile displacement in the perpendicular direction to the initial crack plane for all designs (Figure 5e) and constraining all active structural degrees of freedom at the other end (Figure 5f). We meshed the DCB models with the quadratic tetrahedral (C3D10) element shown in Figure 4g, and then the mesh independency analysis was performed.

\subsection{SLS test}

The SLS specimens were manufactured by FDM according to ASTM D3136 and based on its recommended dimensions shown in Figure 6a. Manufactured specimens were pulled apart using the Universal Testing Machine (Zwick, GA, USA) in tension, equipped with a $25 \mathrm{kN}$ load cell (Figure 6b). The crosshead speed was $1.3 \mathrm{~mm} / \mathrm{min}$, and we tested five specimens for each design. Based on ASTM D3136, the grip must be a 1 by 1-inch square, and we tightened it sufficiently to prevent slipping during testing. 
To simulate the behavior of the SLS test of three different designs and extract cohesive parameters in Mode II, we assigned the isotropic elastic material model for PLA to all models similar to the DCB test. We simulated the boundary condition of the SLS test by constraining all active structural degrees of freedom at one end (Figure 6c) and exerting a tensile displacement at another end of the specimen in the longitudinal direction (Figure $6 \mathrm{~d}$ ). The DCB models meshed, with the quadratic tetrahedral (C3D10) element, are shown in Figure 6e, and then the mesh independency analysis was performed.
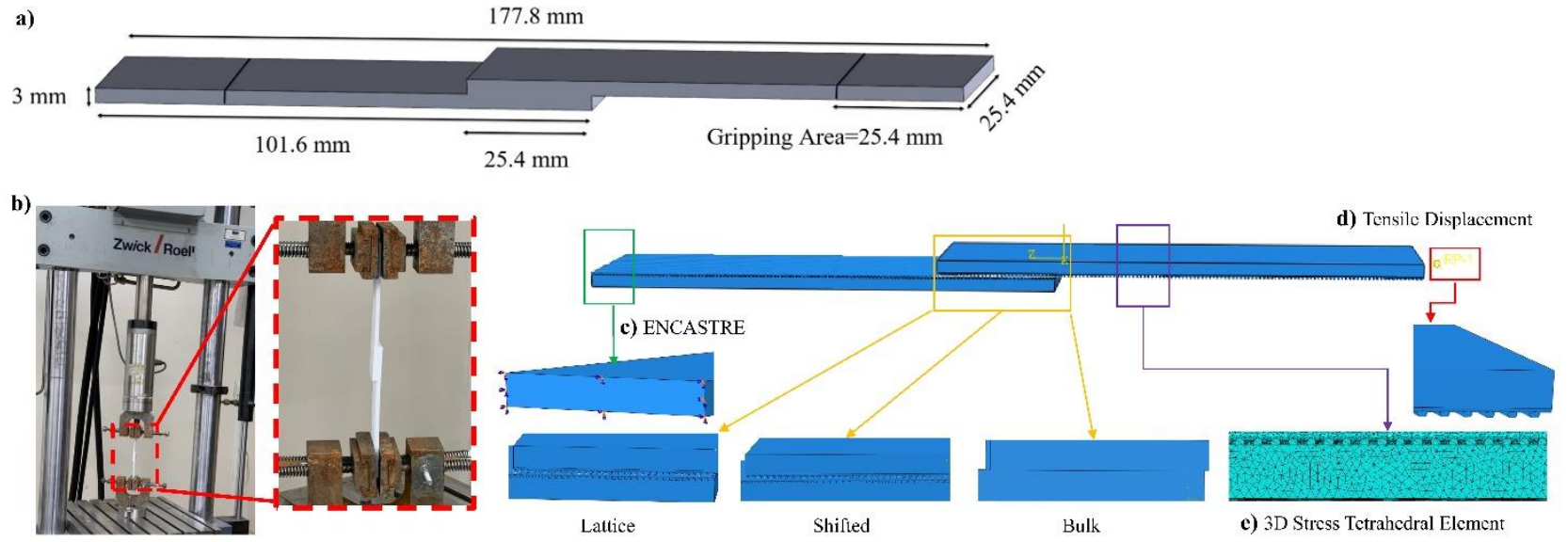

Lattice

Shifted

Bulk

e) 3D Stress Tetrahedral Element

Figure 6. The SLS test specifications in experimental (a \& b) and numerical (c-f) studies. (a) The specimen dimensions based on ASTM D3136, (b) Performing SLS test, (c) Constraining all degree of freedom at the end of the specimen, (d) Tensile displacement to simulate loading regime, (e) Using quadratic tetrahedral (C3D10) elements for meshing of Lattice, Shifted and Bulk designs.

To obtain the bonding parameters, we used the CZM for the numerical simulation of a DCB and SLS tests for Modes I and II, respectively, and implemented in the ABAQUS 2018 (Dassault Systemes Simulia Corp, France) FEM package. The surface-to-surface contact with the definition 
of damage initiation and propagation was assigned to adjacent layers. Cohesive behavior was characterized by assigning the value of initial stiffness $\left(K_{n}, K_{s}, K_{t}\right)$ to the model. Moreover, to optimize the initial stiffness of Mode II, $K_{n}$ was calculated from the DCB test; $K_{s}$ and $K_{t}$ were considered equally. Furthermore, the mechanism of damage was simulated by defining the damage initiation criterion and the damage evolution law.

The damage initiation is the start of the cohesive response degradation at a contact point. In this paper, we have employed the maximum nominal stress criterion as a damage initiation criterion (Equation 4).

$$
\max \left\{\frac{t_{n}}{t_{n}^{0}}, \frac{t_{s}}{t_{s}^{0}}, \frac{t_{t}}{t_{t}^{0}}\right\}=1
$$

Where $t_{n}^{0}, t_{s}^{0}$ and $t_{t}^{0}$ are the peak values of the contact stress when the debonding is either purely normal to the interface or in the first or the second shear direction, respectively. As the contact stresses $\left(t_{n}, t_{s}, t_{t}\right)$ reach certain criteria of damage initiation, the degradation process begins. Moreover, to optimize the cohesive strength of Mode II, we calculated $T_{n}$ from the DCB test and considered $T_{s}$ and $T_{t}$ equally. After reaching the initiation criterion, the cohesive stiffness is degraded base on damage evolution law. This law can be described by fracture energy (G), which is the energy dissipation because of the damage process. We assume the viscosity coefficient of 0.0002 for damage stabilization to facilitate solution convergence. Finally, an inverse analysis procedure was started by initial guesses of CZM parameters and continued until appropriate parameters to fit the experimental data were obtained. Besides, to distinguish each cohesive parameter, the other two parameters were kept constant in FEM simulations.

\subsection{Morphology characterization}


The morphology of specimens was evaluated using scanning electron microscopy (SEM) (AIS2100, SERON Technology, South Korea). First, using a sputter-coater, the samples were goldcoated. Then, by evaluating SEM images, the strut diameter, and the pore size of the fabricated scaffolds were measured.

\section{Results}

\subsection{D printing and characterization:}

The samples were 3D printed with specified parameters in Figure 7, and SEM images were captured from top and side views. According to this image, two different scaffold designs have rectangular-shaped axial and lateral interconnected pores. The average strut diameter, pore size from top view for porous designs, and layer height were measured from SEM images and presented in Figure 7.
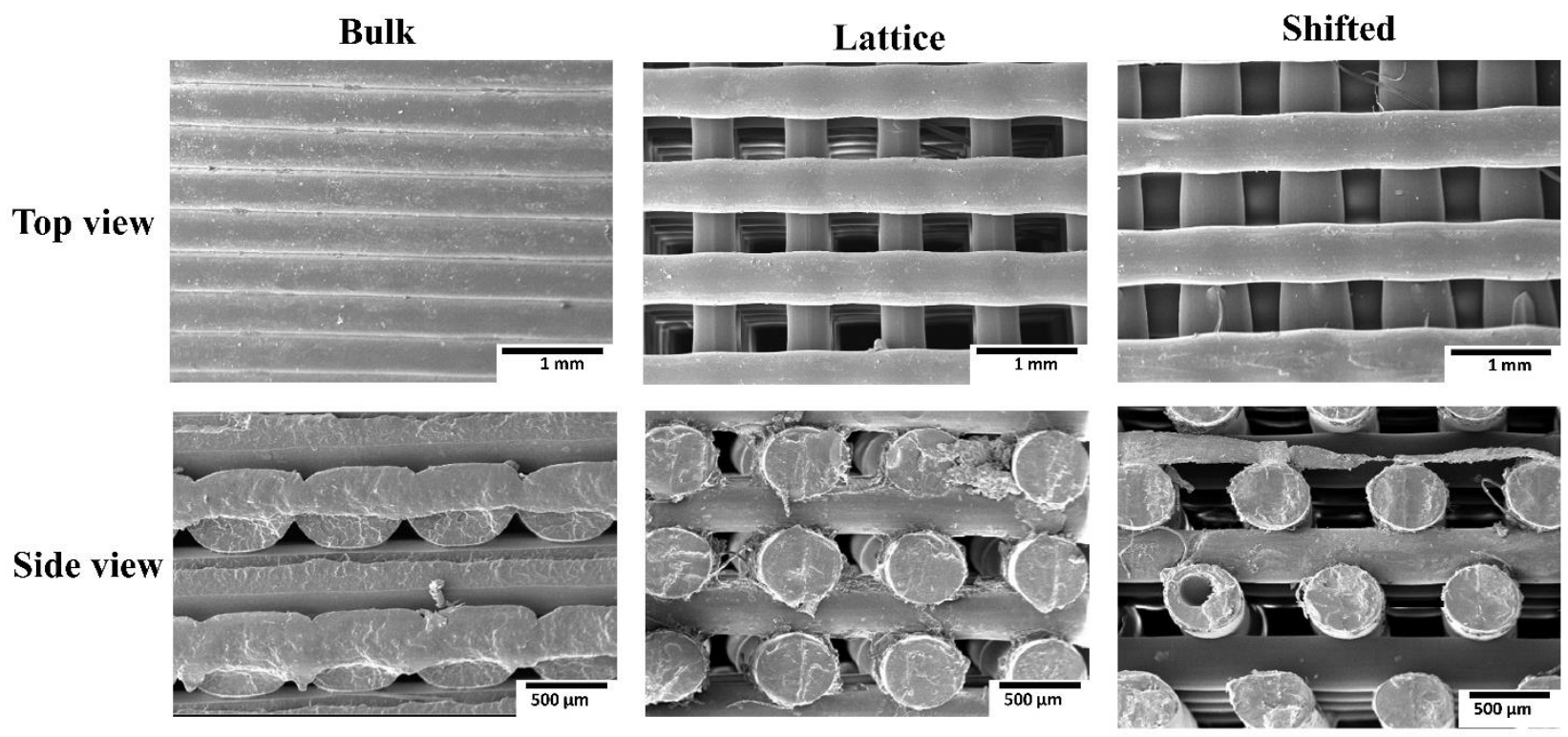

\begin{tabular}{|c|c|}
\hline Layer thickness $(\mathrm{mm})$ & 0.3 \\
\hline Printing speed $(\mathrm{mm} / \mathrm{s})$ & 30 \\
\hline Extrude flow rate & $100 \%$ \\
\hline Bed temperature $\left({ }^{\circ} \mathrm{C}\right)$ & 50 \\
\hline Nozzle temperature $\left({ }^{\circ} \mathrm{C}\right)$ & 195 \\
\hline
\end{tabular}

\begin{tabular}{|c|c|c|c|}
\hline Design & Strut Diameter $(\mu \mathrm{m})$ & Pore Size $(\mu \mathrm{m})$ & Layer Height $(\mu \mathrm{m})$ \\
\hline Lattice & $413.96 \pm 13.58$ & $378.53 \pm 21.40$ & $319.15 \pm 3.46$ \\
\hline Shifted & $407.65 \pm 15.80$ & $430.17 \pm 24.77$ & $327.68 \pm 6.32$ \\
\hline Bulk & $409.83 \pm 21.33$ & - & $326.22 \pm 10.24$ \\
\hline
\end{tabular}


Figure 7. 3D printing parameters, SEM images of Bulk, Lattice, and Shifted samples from top and side views, and morphological parameters of strut diameter, pore size, and layer height measured from SEM images.

It is noticeable that in the printed models, there were more extruded materials in comparison to their computational models, specifically in contact regions between layers. It is more evident in the Bulk model in Figure 7 from the side view compare to its CAD model in Figure 3. In its printed sample, there were lateral contact areas between filaments, while these areas did not exist in the designed model.

\subsection{DCB and SLS tests:}

The experimental and numerical processes to analyze the DCB and SLS tests for obtaining the cohesive parameters in Modes I and II are depicted in Figures 8 and 9, respectively. Also, the representative load-displacement curves of the specimens and the predicted numerical results by variation of cohesive parameters are shown in these figures. In both DCB and SLS tests, the load increment is linear relatively with displacement increment until the crack initiation. Then, the load increased at a nonlinear decreasing rate during the crack growth. When it reaches a maximum value, unstable and unexpected crack growth leads to the ultimate failure. As shown in Figure 8, the Bulk sample needed more crosshead displacement and fracture energy to complete the DCB test compared to porous designs. However, the trend of load increasing with displacement increment was pretty similar in all designs. 

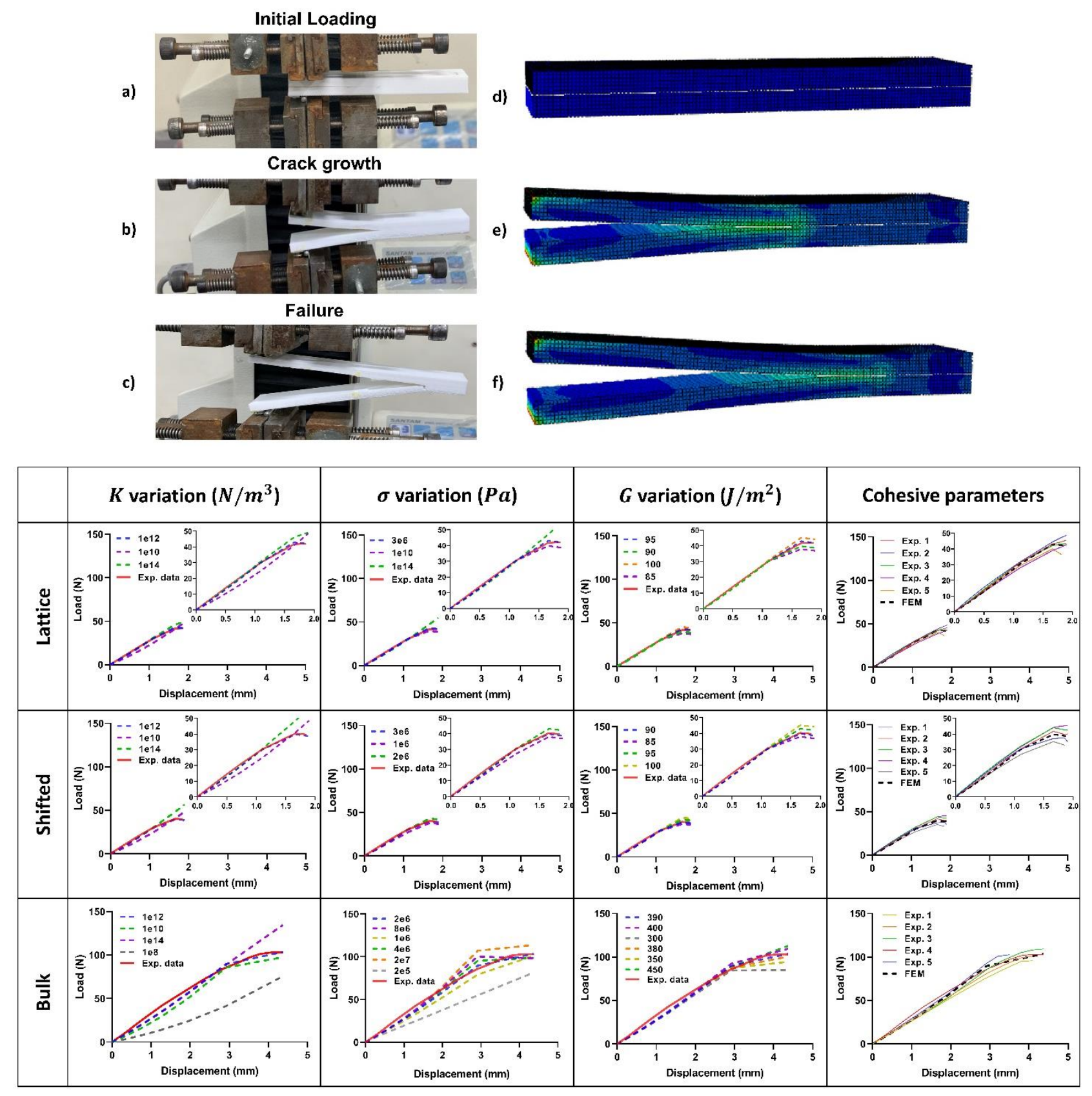

Figure 8. The double cantilever beam (DCB) test steps and its computational simulation. The loaddisplacement curves with the variation of cohesive parameters were compared with experimental data, and the best fitting was selected. In the right column, the numerical results with selected cohesive parameters and 5 DCB test results are illustrated. 
Initial Loading

a)

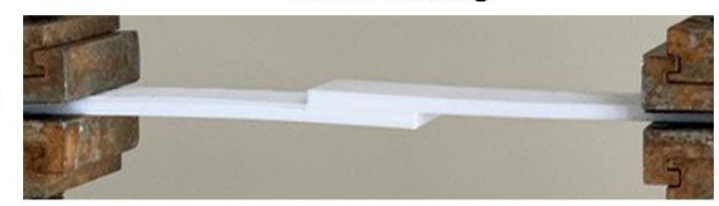

Bending Moment

b)

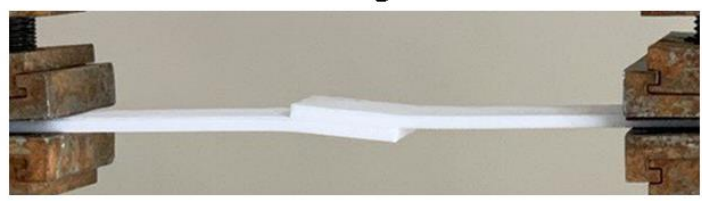

Failure

c)

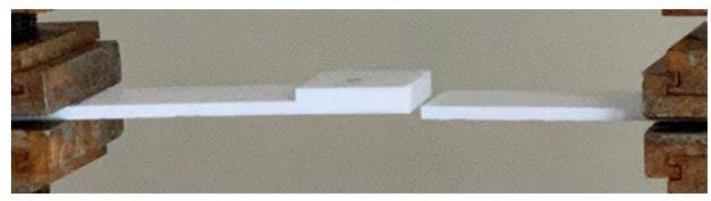

d)

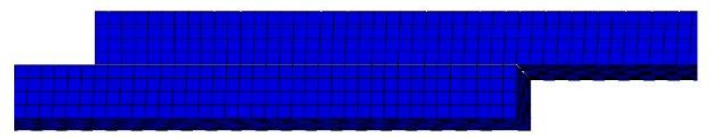

e)

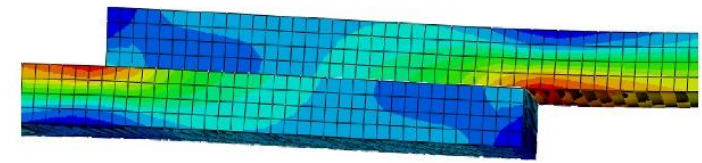

f)

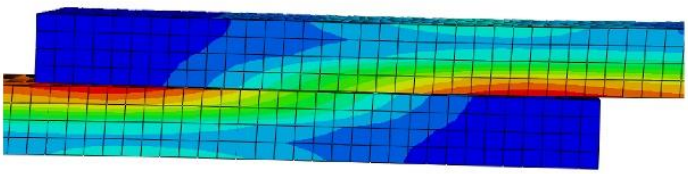

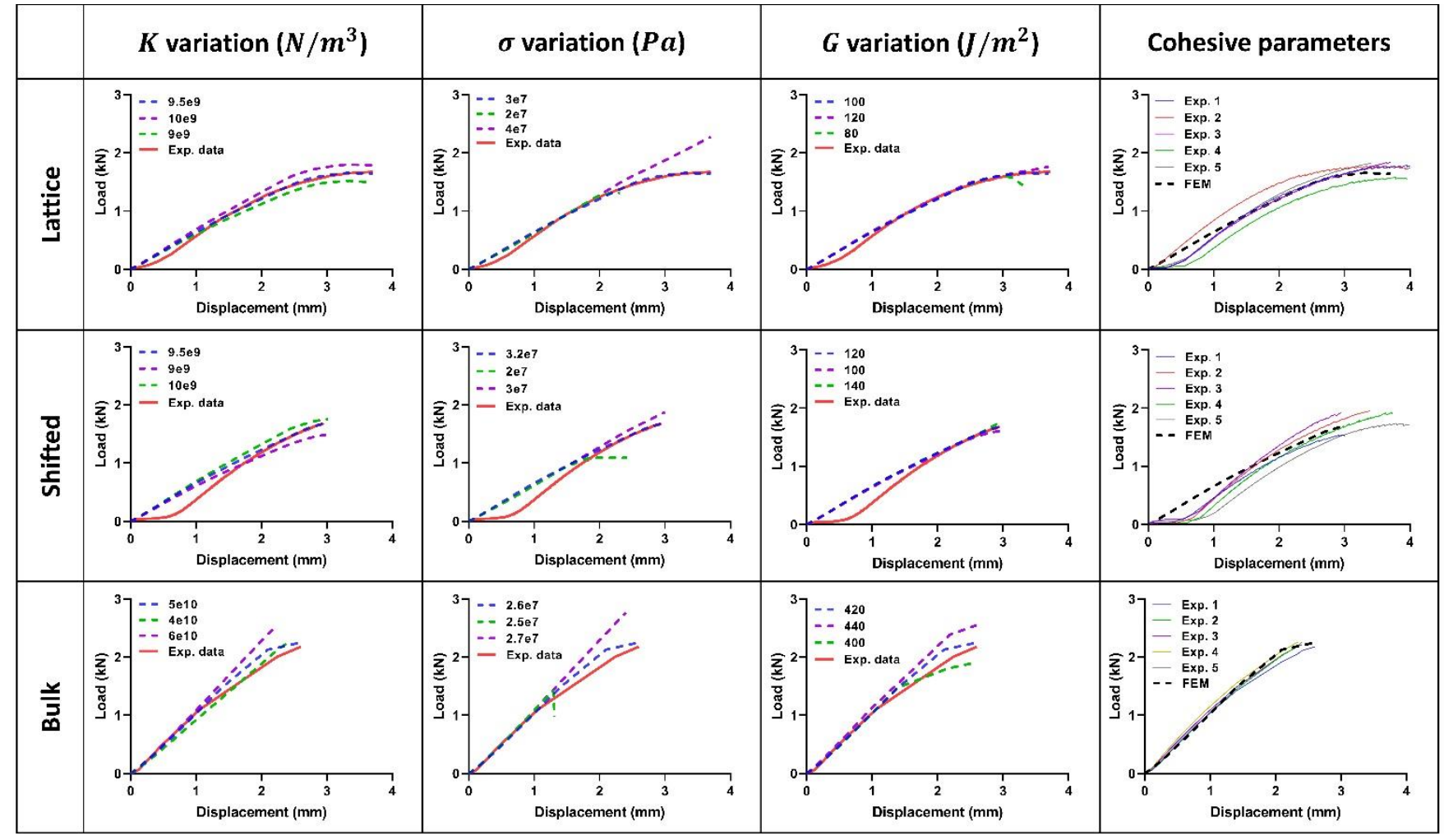

Figure 9. The single lap shear (SLS) test steps (a-c) and its computational simulation (d-f). The load-displacement curves with the variation of cohesive parameters were compared with 
experimental data, and the best fitting was selected. In the right column, the numerical results with selected cohesive parameters and 5 SLS test results are illustrated.

As shown in Figure 9, the Bulk sample showed more stiffness than porous designs in the SLS test. However, there was no significant difference between total displacement in all designs in contrast to the DCB test. At the maximum loading level, we simulated the high distortion of the substrates using the FEM model (Figures 9a, b, d, e), and the debonding propagation in adhesive layers interfaces as clearly illustrated in Figures $9 \mathrm{e}$ and $9 \mathrm{f}$ agreed with experimental curves.

In general, the Bulk model showed more resistance to debonding than porous designs as its failure occurred in higher fracture energies. Besides, there were no significant differences between the mechanical behavior of Lattice and Shifted designs during DCB and SLS tests from load-displacement curves and cohesive parameters. The cohesive parameters in Modes I and II of three different designs extracted from DCB and SLS tests are represented in Table 1.

Table 1. The cohesive parameters: initial stiffness (K), cohesive strength $(T)$, and fracture energy (G) in Modes I and II of Bulk, Lattice, and Shifted designs

\begin{tabular}{|c|c|c|c|c|c|c|}
\cline { 2 - 7 } \multicolumn{1}{c|}{} & $\boldsymbol{K}_{\boldsymbol{n}}\left(\mathrm{N} / \mathrm{m}^{3}\right)$ & $\boldsymbol{K}_{\boldsymbol{s}}=\boldsymbol{K}_{\boldsymbol{t}}\left(\mathrm{N} / \mathrm{m}^{3}\right)$ & $\boldsymbol{T}_{\boldsymbol{n}}(\boldsymbol{P a})$ & $\boldsymbol{T}_{\boldsymbol{s}}=\boldsymbol{T}_{\boldsymbol{t}}(\boldsymbol{P a})$ & $\boldsymbol{G}_{\boldsymbol{I}}\left(\boldsymbol{J} / \mathrm{m}^{2}\right)$ & $\boldsymbol{G}_{\boldsymbol{I I}}\left(\boldsymbol{J} / \mathrm{m}^{2}\right)$ \\
\hline Bulk & $1.0 e^{12}$ & $5.0 e^{10}$ & $2.0 e^{6}$ & $2.6 e^{7}$ & $3.9 e^{2}$ & $4.2 e^{2}$ \\
\hline Lattice & $1.0 e^{12}$ & $9.5 e^{9}$ & $3.0 e^{6}$ & $3.0 e^{7}$ & $9.5 e^{1}$ & $1.0 e^{2}$ \\
\hline Shifted & $1.0 e^{12}$ & $9.5 e^{9}$ & $3.0 e^{6}$ & $3.2 e^{7}$ & $9.0 e^{1}$ & $1.2 e^{2}$ \\
\hline
\end{tabular}

As it shows in Table 1, the initial stiffness of all designs was similar in Mode I but was around five times for Bulk in comparison to porous designs in this Mode. Moreover, the cohesive strength of 
the Bulk model in Modes I and II is slightly lower than the porous designs. However, the fracture energy in both Modes for the Bulk model is around four times of two others.

\subsection{Cohesive parameters utilization:}

The comparison between the assumption of cohesive contact between layers of specimens and fully bonded contact using tie constraint in the software is illustrated in Figure 10. The cohesive assumption improved the accuracy of the computational simulation for all designs, obviously in both DCB and SLS tests. So, it is essential to suppose partially bonded layers using CZM in additive manufactured parts in Modes I and II to simulate void and gaps between layers.

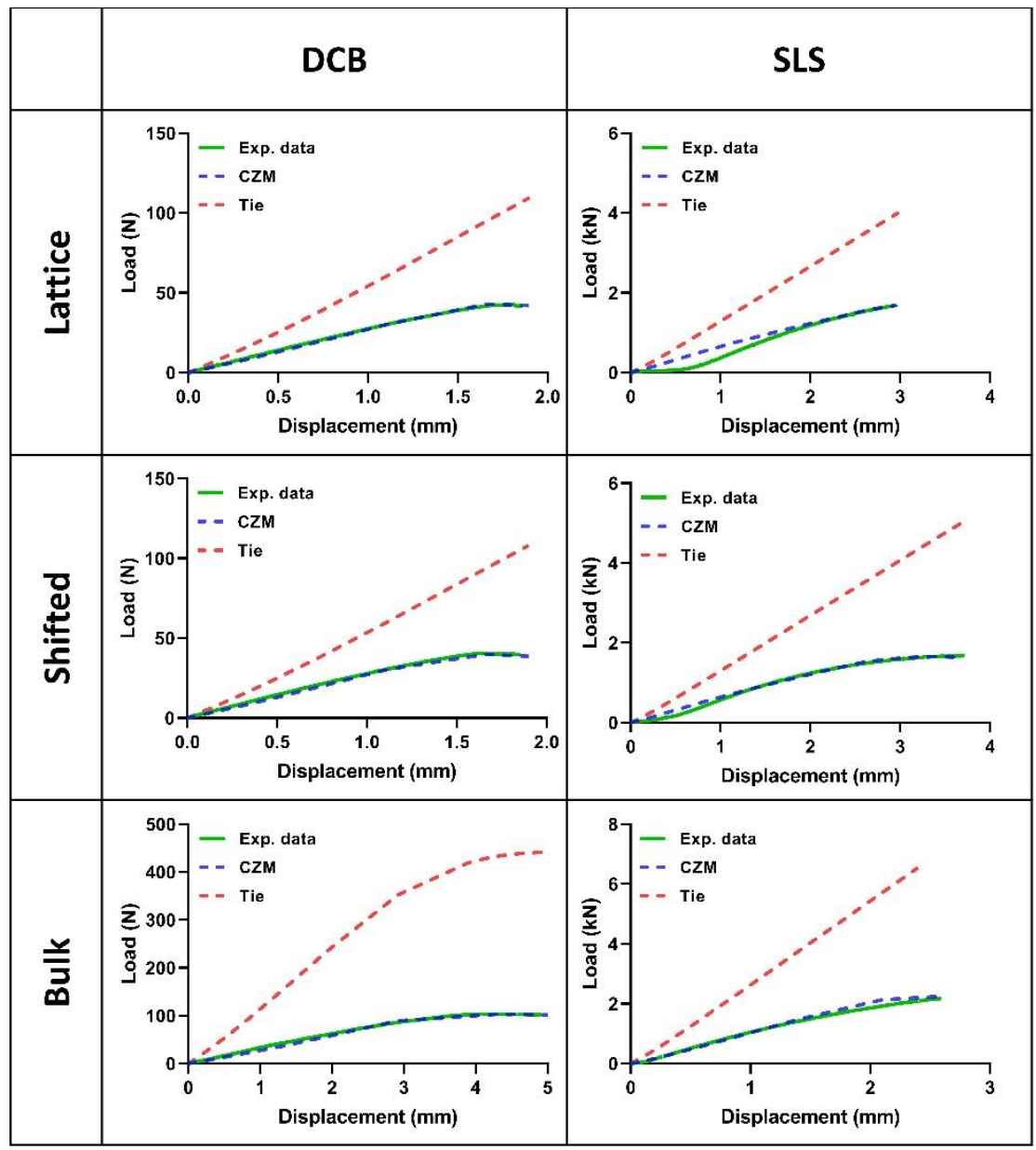


Figure 10. The effect of assuming partially bonded layers by the cohesive zone model (CZM) to simulate interlayer contact for Bulk and porous additive manufacturing parts in comparison to fully bonded (tie constraint in Abaqus software) layers assumption. The modeling of voids and gaps between layers enhanced the computational accuracy using CZM in both Modes I and II.

\section{Discussion}

In the present study, we calculated the cohesive parameters of bonding layers in Mode I and II by comparing the load-displacement curves from numerical simulations and experiments through inverse FEM. The samples were 3D printed with acceptable geometrical accuracy in terms of strut diameter, pore size, and layer height, similar to design assumptions.

There was a reasonable consistency between bilinear law and the experimental data, and they showed similar initial slopes, maximum loads, and trends. The numerical curves were in the domain of experimental curves indicated that the CZM could simulate the crack initiation and propagation of 3D printed scaffolds. In other words, the numerical predictions were in good agreement with experimental results.

Moreover, we had assessed the susceptibility of the simulated mechanical responses to cohesive parameters, i.e., initial stiffness $(K)$, cohesive strength $(T)$, and cohesive energy $(G)$. The initial stiffness affects the slope of the force-displacement curve to reach the maximum strength before damage initiation. Moreover, the initial stiffness value should be large enough to prevent layer penetration but not too large to cause the numerical simulation failure to match experimental data because it may cause fictitious compliance before crack growth. 
Furthermore, by employing different cohesive strengths and energies, the initial slope of the load-displacement curve remained unchanged. However, by energy increment, the maximum load increased, and it led to a higher displacement. Moreover, the cohesive strength is associated with the peak value of the load-displacement curve. The maximum load extremely increased as the cohesive strength increased, but the cohesive strength showed a small effect on the displacement related to the maximum load, in contrast to the cohesive energy.

In Lattice and Shifted designs, because of the equal number of filaments that can carry the load and so the same cohesive surfaces, cohesive parameters that are extracted by the DCB and SLS tests are approximately equal. It can be concluded that the microstructure may not affect the cohesive parameters of the porous design in the same printing conditions.

FEM can simulate the initiation, propagation of the crack, and failure of the DCB test. The crack initiation occurs at about $3 \mathrm{~mm}$ displacement in Bulk specimens, while at about $1.5 \mathrm{~mm}$ in Lattice and Shifted specimens, indicating that there was more resistance to debonding in the Bulk sample. Also, despite the similar values of initial stiffness and cohesive strength, the fracture energy was around four times for the Bulk model compared to porous designs that can be due to the difference between FEM and printed model. The FEM model was an ideal model with simplified geometry, and the excess material deposited in printed samples, especially in contact regions of struts, didn't exist in it. So, the contact region in the printed specimen enhanced in comparison to the FEM model for Bulk design, as showed in Figure 7 (Bulk model, side view). However, the porosity of Lattice and Shifted microstructures can be influential on the level of fracture energy between 3 designs, and the non-porous microstructure of the Bulk model can increase this energy. Moreover, the stress distribution during initial bonding, crack initiation, and 
progression was simulated in different steps of the DCB test using FEM, which was not attainable using experimental approaches.

The FEM model can model the excessive distortion of the substrates at the peak loading level produced by the huge eccentricity of the adhesive layers (Figure 9b) in the SLS test simulation. Moreover, in agreement with experimental findings, this method can simulate the debonding propagation in the adhesive layers interface.

The initial stiffness for the Bulk model in the SLS test was around five times higher than porous designs that can be due to porosity or strong lateral bonding between struts of the Bulk model because of excess material deposition as observed in Figure 7 (Bulk model, side view). Moreover, the fracture energy of the Bulk sample was around four times of porous designs in SLS tests like the DCB test. It can be because of the higher contact region in printed samples compared to the FEM model or non-porous microstructure as stated before for the DCB test. Furthermore, there was a softening region in the initial load-displacement of the Lattice and Shifted designs SLS experimental test, which did not exist in the numerical curve that can be due to simplification in the FEM model and the lower amount of porous layers in comparison to actual 3D printed samples.

It is noticeable that we proved that the assumption of cohesive contact between layers was essential for the FEM analysis of the 3D printed parts in Modes I and II, as seen in Figure 10. In other words, there was a significant difference between full and partial bonding predictions to fit experimental data. Furthermore, this inverse FEM method can be further employed to predict the bond formation in 3D printed products, and the CZM parameters derivation under different manufacturing conditions because the printing parameters affect the properties of scaffold [5]. 
We note that the developed FEM models introduced in this study did not fully represent the actual microstructure of 3D printed samples, and it can affect strength and fracture properties $[3,4,43]$. More realistic models with exact porous layer numbers and interlayer contact regions can further improve the predictions and can be pursued in future studies.

In reality, there is no generation of excessive molding pressure and shear rate in the FDM process opposite to the relevant extrusion and injection thermoplastic processing routes. Hence, it is impossible to develop appropriate cohesion with strong interactions between layers. Indeed, in this printing method, the filament adhesion is mainly because of interdiffusion phenomena that can control the mechanical response. Accordingly, this novel technology facilitates the manufacture of parts with complicated geometry [1] but with moderate bonding properties that can be improved by post-processing procedures, e.g., heating [6]. Therefore, using CZM in Mode I and II is essential to predict the mechanical properties because of this partial bonding between layers of FDM parts

In this study, we measured the bonding strength of 3D printed PLA scaffolds using CZM and inverse FEM in Modes I and II, and investigated the effect of porosity and microstructure. The cohesive parameters of porous designs were the same in Modes I and II which means that these parameters can be used for other porous designs, printed with the PLA in similar printing conditions. Also, the computational simulations illustrated the stress distribution in the crack propagation, and it means that the unmeasurable quantities in experiments can be estimated using numerical models. Moreover, results showed that it is essential to consider the partial bonding between layers for simulating the mechanical properties of 3D printed samples, especially in fracture Modes I and II. The work demonstrated that the framework of CZM and 
FEM methodology could be implemented to estimate the adhesion between layers of the 3D printed parts, which lead to more realistic and accurate mechanical properties predictions.

\section{Declarations:}

Funding: This research did not receive any specific grant from funding agencies in the public, commercial, or not-for-profit sectors.

Conflicts of interest /Competing interests: The authors have no relevant financial or nonfinancial interests to disclose.

Availability of data and material: Yes, the data and material are available.

Code availability: Yes, the code is available.

Authors' contributions: Nogol Nazemzadeh: Investigation, Formal analysis, Software, Writing Original Draft, Data Curation Anahita Ahmadi Soufivand: Conceptualization, Methodology, Draft Review \& Editing, Visualization, Supervision Nabiollah Abolfathi: Conceptualization, Resources, Project administration

Ethics approval: Not applicable

Consent to participate: Not applicable

Consent for publication: Not applicable

\section{Reference}

1. Soufivand, A.A., et al., Prediction of mechanical behavior of 3D bioprinted tissue-engineered scaffolds using finite element method (FEM) analysis. Additive Manufacturing, 2020: p. 101181.

2. Koch, C., L. Van Hulle, and N. Rudolph, Investigation of mechanical anisotropy of the fused filament fabrication process via customized tool path generation. Additive Manufacturing, 2017. 16: p. 138-145.

3. Standard, A., Standard terminology for additive manufacturing technologies. ASTM International F2792-12a, 2012.

4. Isakov, D., et al., 3D printed anisotropic dielectric composite with meta-material features. Materials \& Design, 2016. 93: p. 423-430. 
5. Soufivand, A.A., et al., The effect of 3D printing on the morphological and mechanical properties of polycaprolactone filament and scaffold. Polymers for Advanced Technologies, 2020. 31(5): p. 1038-1046.

6. Naghieh, S., et al., Numerical investigation of the mechanical properties of the additive manufactured bone scaffolds fabricated by FDM: the effect of layer penetration and post-heating. Journal of the mechanical behavior of biomedical materials, 2016. 59: p. 241-250.

7. Naghieh, S., et al., Fused deposition modeling and fabrication of a three-dimensional model in maxillofacial reconstruction. Regeneration, Reconstruction \& Restoration, 2016. 1(3): p. 139-144.

8. Boccardi, E., et al., Oxygen diffusion in marine-derived tissue engineering scaffolds. Journal of Materials Science: Materials in Medicine, 2015. 26(6): p. 200.

9. Sherwood, J.K., et al., A three-dimensional osteochondral composite scaffold for articular cartilage repair. Biomaterials, 2002. 23(24): p. 4739-4751.

10. Felfel, R., et al., In vitro degradation and mechanical properties of PLA-PCL copolymer unit cell scaffolds generated by two-photon polymerization. Biomedical Materials, 2016. 11(1): p. 015011.

11. Serra, T., et al., Relevance of PEG in PLA-based blends for tissue engineering 3D-printed scaffolds. Materials Science and Engineering: C, 2014. 38: p. 55-62.

12. Tyler, B., et al., Polylactic acid (PLA) controlled delivery carriers for biomedical applications. Advanced Drug Delivery Reviews, 2016. 107: p. 163-175.

13. Senatov, F.S., et al., Mechanical properties and shape memory effect of 3D-printed PLA-based porous scaffolds. Journal of the mechanical behavior of biomedical materials, 2016. 57: p. 139148.

14. Tayton, E., et al., A comparison of polymer and polymer-hydroxyapatite composite tissue engineered scaffolds for use in bone regeneration. An in vitro and in vivo study. Journal of Biomedical Materials Research Part A, 2014. 102(8): p. 2613-2624.

15. Grémare, A., et al., Characterization of printed PLA scaffolds for bone tissue engineering. Journal of Biomedical Materials Research Part A, 2018. 106(4): p. 887-894.

16. Martínez, J., et al., Comparative between FEM models for FDM parts and their approach to a real mechanical behaviour. Procedia Engineering, 2013. 63: p. 878-884.

17. Park, S.-i., N. Watanabe, and D.W. Rosen, Estimating failure of material extrusion truss structures based on deposition modeling and a cohesive zone model. Materials \& Design, 2018. 147: p. 122133.

18. Wang, J., et al., A novel approach to improve mechanical properties of parts fabricated by fused deposition modeling. Materials \& Design, 2016. 105: p. 152-159.

19. Barenblatt, G.I., The mathematical theory of equilibrium cracks in brittle fracture. Advances in applied mechanics, 1962. 7(1): p. 55-129.

20. Dugdale, D.S., Yielding of steel sheets containing slits. Journal of the Mechanics and Physics of Solids, 1960. 8(2): p. 100-104. 
21. Turon, A., et al., An engineering solution for mesh size effects in the simulation of delamination using cohesive zone models. Engineering fracture mechanics, 2007. 74(10): p. 1665-1682.

22. Camanho, P.P., C.G. Davila, and M. De Moura, Numerical simulation of mixed-mode progressive delamination in composite materials. Journal of composite materials, 2003. 37(16): p. 1415-1438.

23. Schellekens, J. and R. De Borst, Numerical simulation of free edge delamination in graphite-epoxy laminates under uniaxial tension, in Composite Structures. 1991, Springer. p. 647-657.

24. Standard, A., D5528-01, "Standard test method for Mode I Interlaminar Fracture Toughness of Unidirectional Fiber-Reinforced Polymer Matrix Composites," ASTM International, West Conshohocken, PA, 2007, DOI: 10.1520/D5528-94A.

25. Valoroso, N. and R. Fedele, Characterization of a cohesive-zone model describing damage and decohesion at bonded interfaces. Sensitivity analysis and mode-I parameter identification. International Journal of Solids and Structures, 2010. 47(13): p. 1666-1677.

26. Gustafson, P.A. and A.M. Waas, The influence of adhesive constitutive parameters in cohesive zone finite element models of adhesively bonded joints. International Journal of Solids and Structures, 2009. 46(10): p. 2201-2215.

27. Maier, G., M. Bocciarelli, and R. Fedele, Some innovative industrial prospects centered on inverse analyses, in Parameter Identification of Materials and Structures. 2005, Springer. p. 55-93.

28. Oh, J.-C. and H.-G. Kim, Inverse estimation of cohesive zone laws from experimentally measured displacements for the quasi-static mode I fracture of PMMA. Engineering Fracture Mechanics, 2013. 99: p. 118-131.

29. Wang, J., et al., Viscoelastic adhesive interfacial model and experimental characterization for interfacial parameters. Mechanics of Materials, 2010. 42(5): p. 537-547.

30. Bocciarelli, M. and G. Bolzon, Indentation and imprint mapping for the identification of interface properties in film-substrate systems. International journal of fracture, 2009. 155(1): p. 1-17.

31. Kishore, V., et al., Infrared preheating to improve interlayer strength of big area additive manufacturing (BAAM) components. Additive Manufacturing, 2017. 14: p. 7-12.

32. Spoerk, M., et al., Parametric optimization of intra-and inter-layer strengths in parts produced by extrusion-based additive manufacturing of poly (lactic acid). Journal of applied polymer science, 2017. 134(41): p. 45401.

33. Seppala, J.E., et al., Weld formation during material extrusion additive manufacturing. Soft Matter, 2017. 13(38): p. 6761-6769.

34. Liravi, F., S. Das, and C. Zhou, Separation force analysis and prediction based on cohesive element model for constrained-surface Stereolithography processes. Computer-Aided Design, 2015. 69: p. 134-142.

35. Ahmadi, A., et al., Effect of manufacturing parameters on mechanical properties of 316L stainless steel parts fabricated by selective laser melting: A computational framework. Materials \& Design, 2016. 112: p. 328-338. 
36. Spackman, C.C., et al., A Cohesive Zone Model for the Stamping Process Encountered During Three-Dimensional Printing of Fiber-Reinforced Soft Composites. Journal of Manufacturing Science and Engineering, 2018. 140(1).

37. Fonseca, J., et al., Study of the interlaminar fracture under mode I loading on FFF printed parts. Composite Structures, 2019. 214: p. 316-324.

38. Kumar, S., B.L. Wardle, and M.F. Arif, Strength and performance enhancement of bonded joints by spatial tailoring of adhesive compliance via 3D printing. ACS applied materials \& interfaces, 2017. 9(1): p. 884-891.

39. Kovan, V., G. Altan, and E.S. Topal, Effect of layer thickness and print orientation on strength of 3D printed and adhesively bonded single lap joints. Journal of Mechanical Science and Technology, 2017. 31(5): p. 2197-2201.

40. Garcia, R. and P. Prabhakar, Bond interface design for single lap joints using polymeric additive manufacturing. Composite Structures, 2017. 176: p. 547-555.

41. Falck, R., et al., AddJoining: A novel additive manufacturing approach for layered metal-polymer hybrid structures. Materials Letters, 2018. 217: p. 211-214.

42. Dugbenoo, E., et al., Enhanced Bonding via Additive Manufacturing-Enabled Surface Tailoring of 3D Printed Continuous-Fiber Composites. Advanced Engineering Materials, 2018. 20(12): p. 1800691.

43. Yeo, M.G. and G.H. Kim, Preparation and characterization of 3D composite scaffolds based on rapid-prototyped $\mathrm{PCL} / \beta$-TCP struts and electrospun $\mathrm{PCL}$ coated with collagen and HA for bone regeneration. Chemistry of materials, 2012. 24(5): p. 903-913.

44. Farah, S., D.G. Anderson, and R. Langer, Physical and mechanical properties of PLA, and their functions in widespread applications-A comprehensive review. Advanced drug delivery reviews, 2016. 107: p. 367-392. 


\section{Traction}

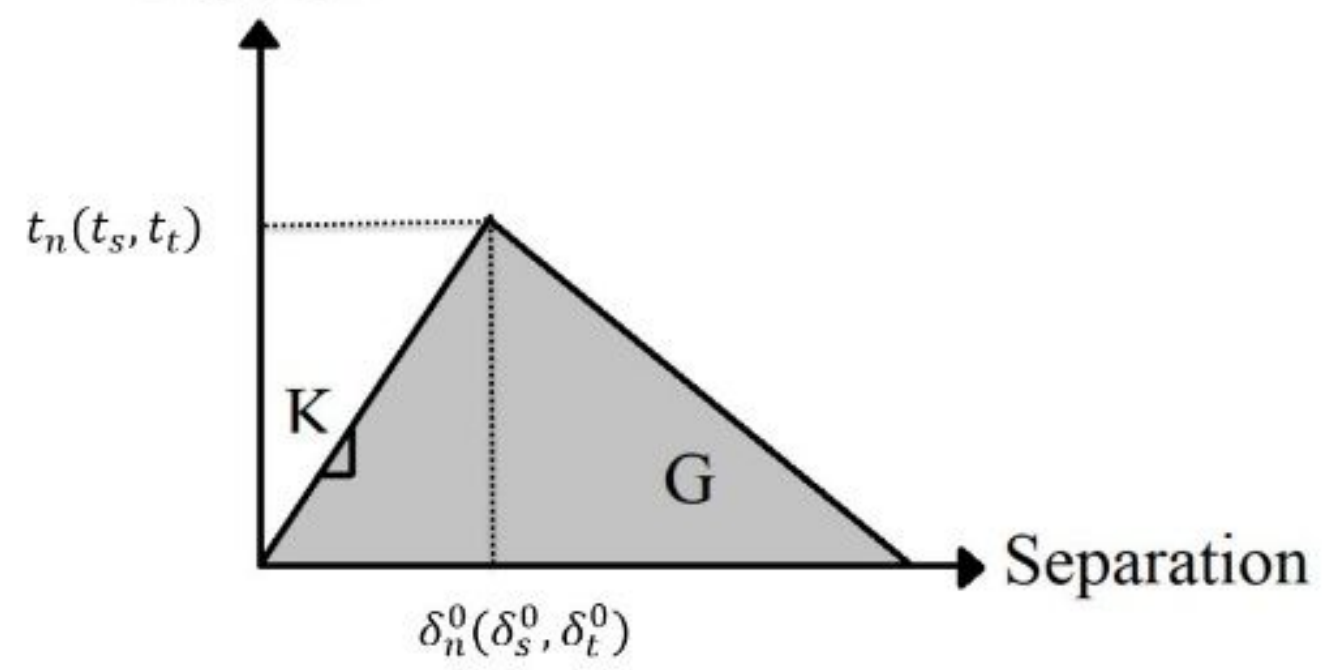

Figure 1

Representation of Cohesive Zone Model (CZM) by linear regions and parameters: initial stiffness $(K)$, cohesive strength $(T)$, and fracture energy $(G)$.

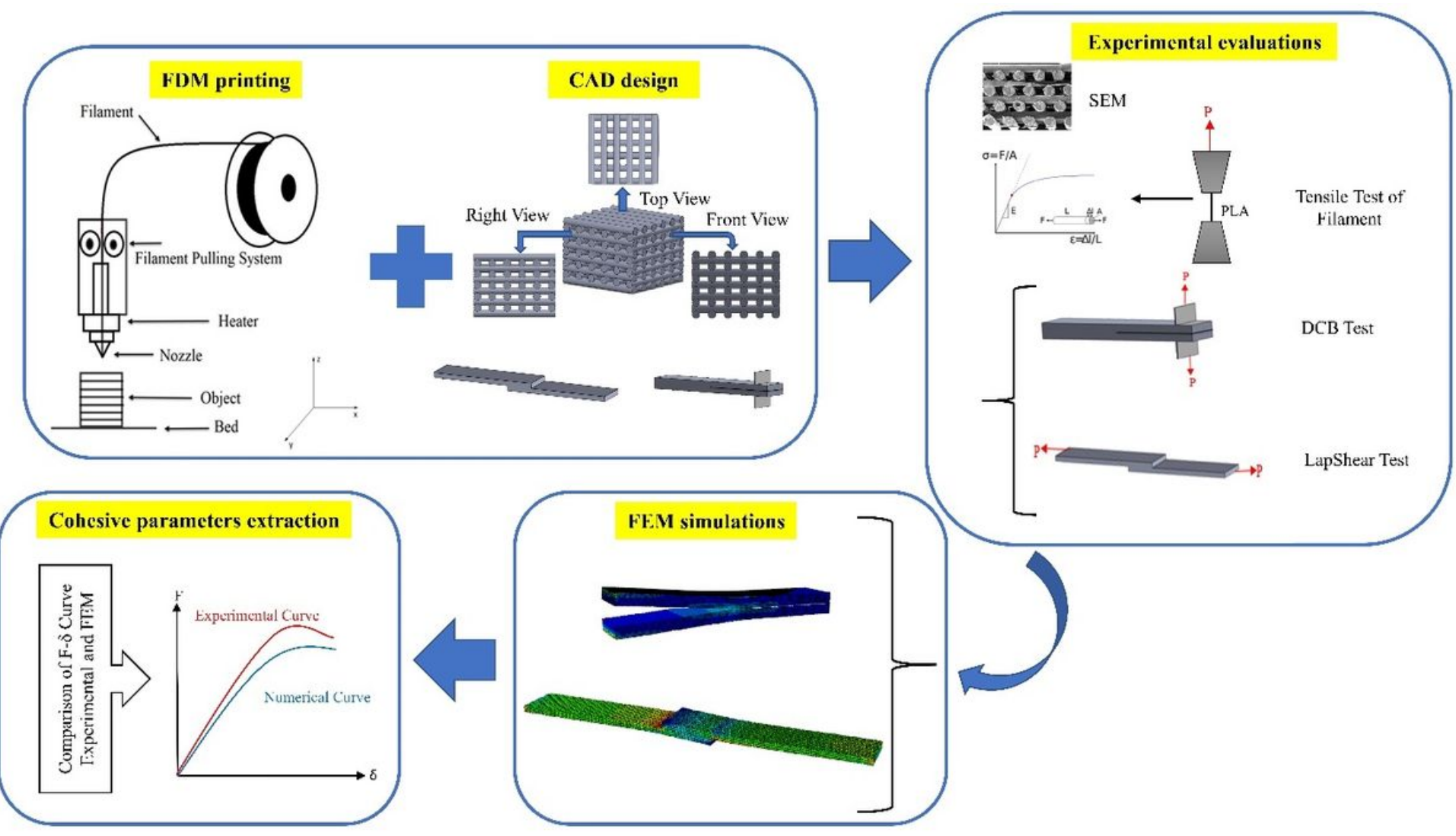

Figure 2 
The research framework was exploited in this study. From the top left, initially, we used the FDM parameters to computer-aided design (CAD) of the 3D printing part. After the manufacturing of specimens, we performed the experimental tests on the filament, DCB, and SLS samples. Then, the FEM simulations with various cohesive parameters were applied, and finally, we extracted the CZM parameters for each design and test by comparing numerical and experimental data.

(a)

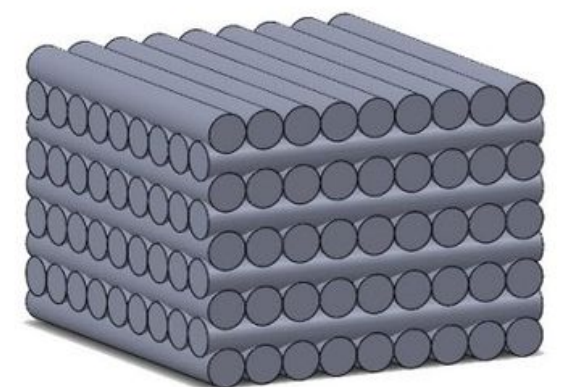

Bulk

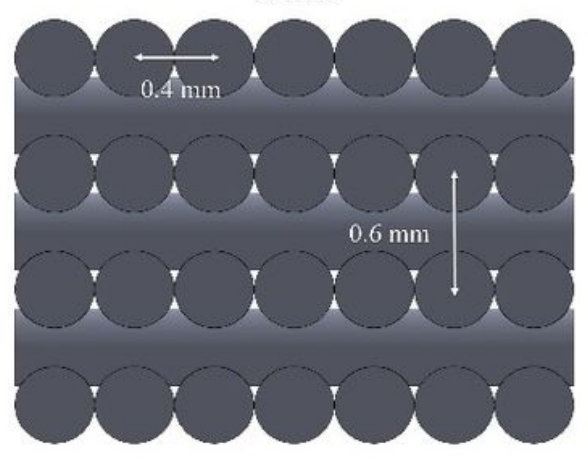

(b)
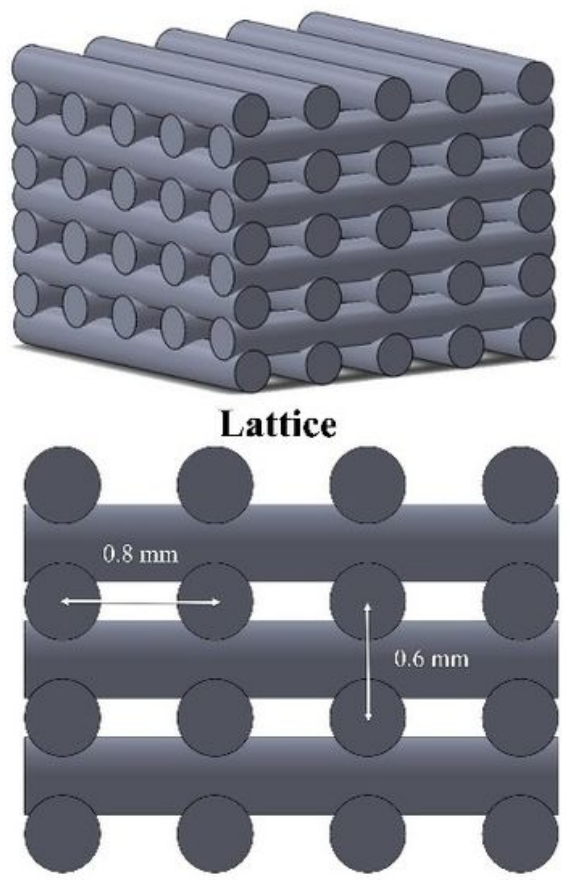

(c)
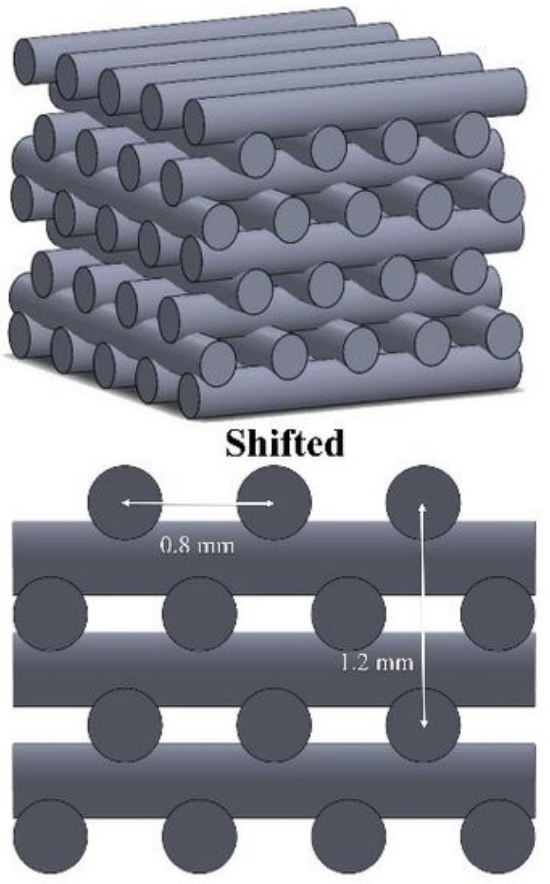

Figure 3

The geometrical specifications of different designs: a) Bulk, b) Lattice, c) Shifted. The FDM parameters were the same for all designs with a strut diameter of $400 \mu \mathrm{m}$ and layer penetration of $100 \mu \mathrm{m}$ as the layer height was $300 \mu \mathrm{m}$. 


\section{Filament preparation}
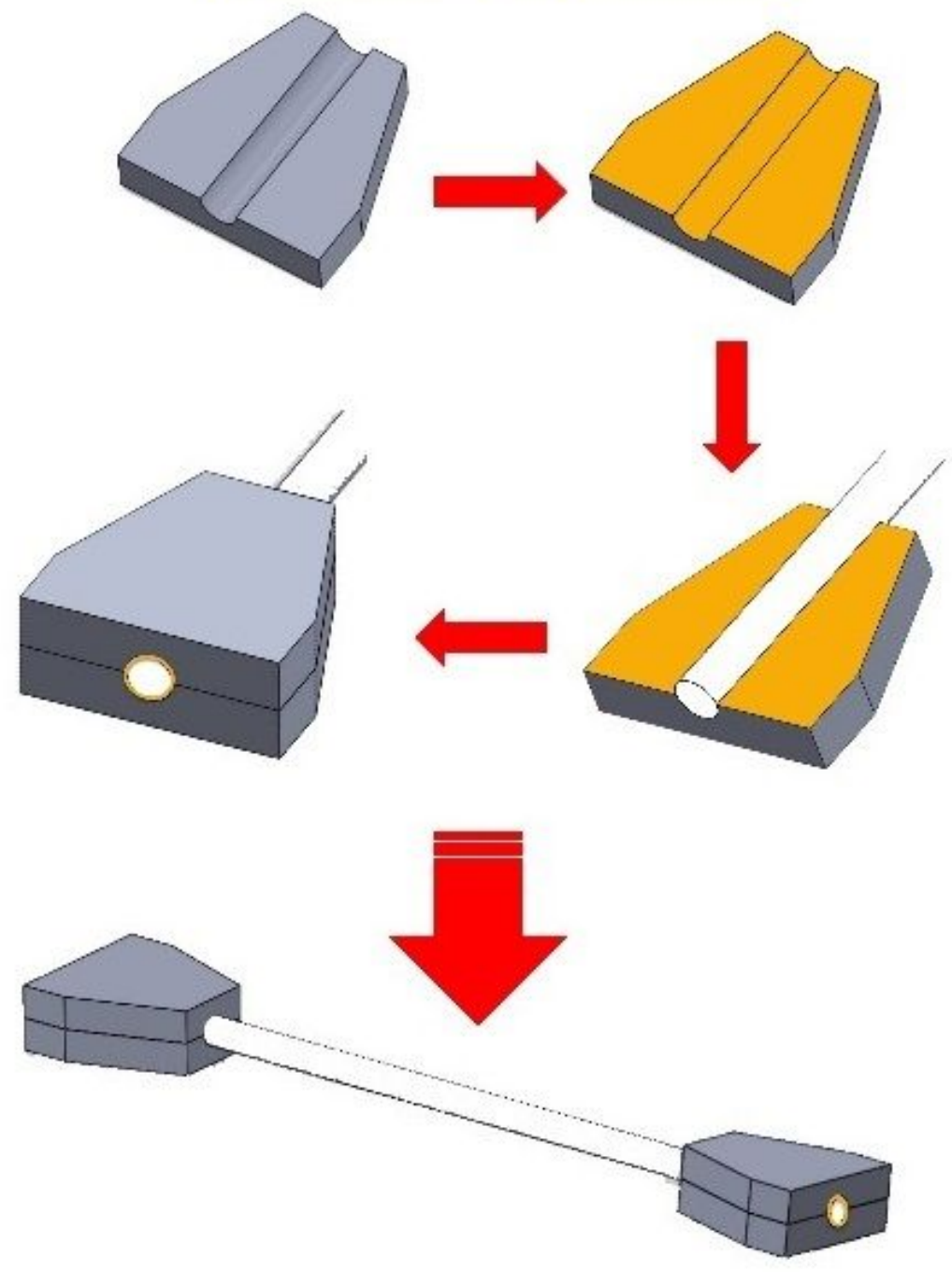

\section{Tensile test setup}

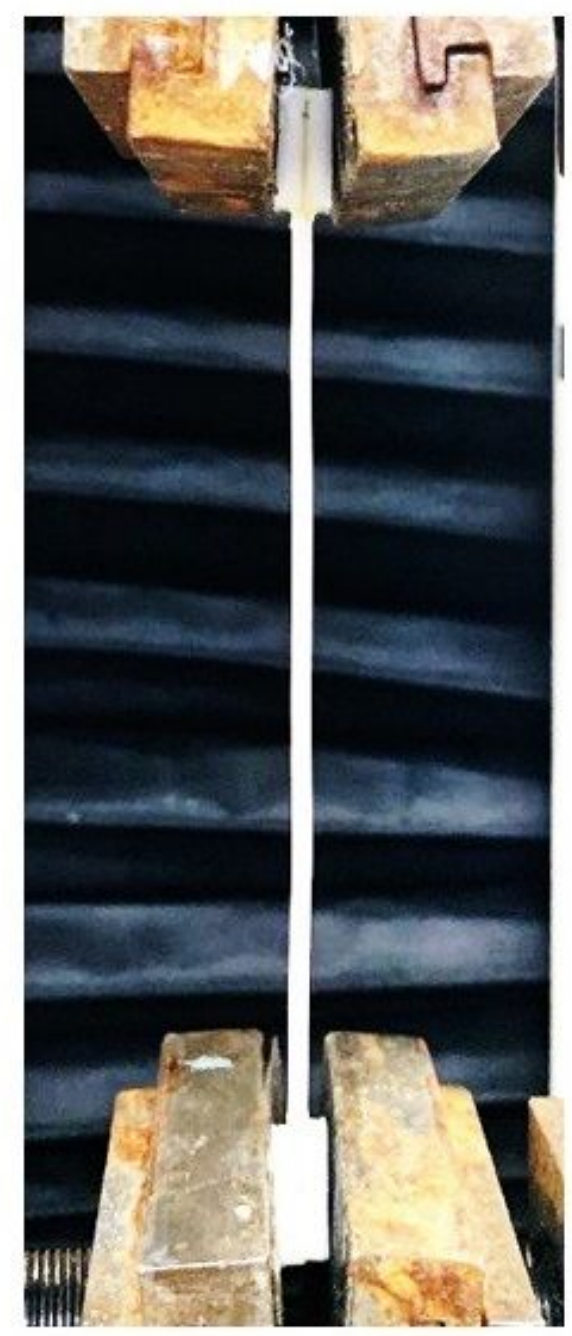

\section{Figure 4}

Tensile test configuration for PLA filament. First, the $3 \mathrm{~d}$ printed PLA dog-bone parts were glued to the filament, and then the tensile test was performed $(n=3)$. 
a)

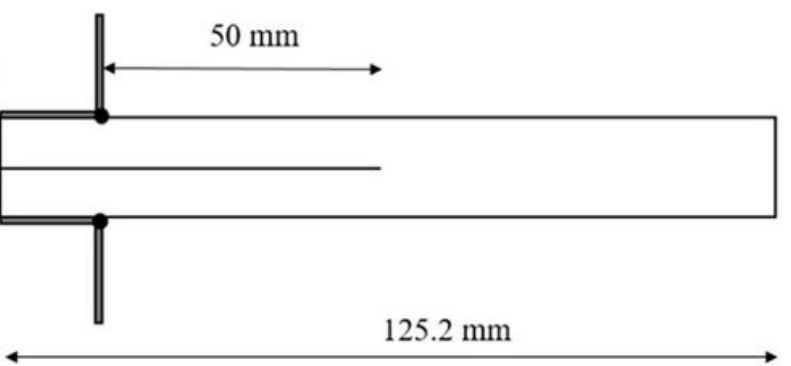

b)

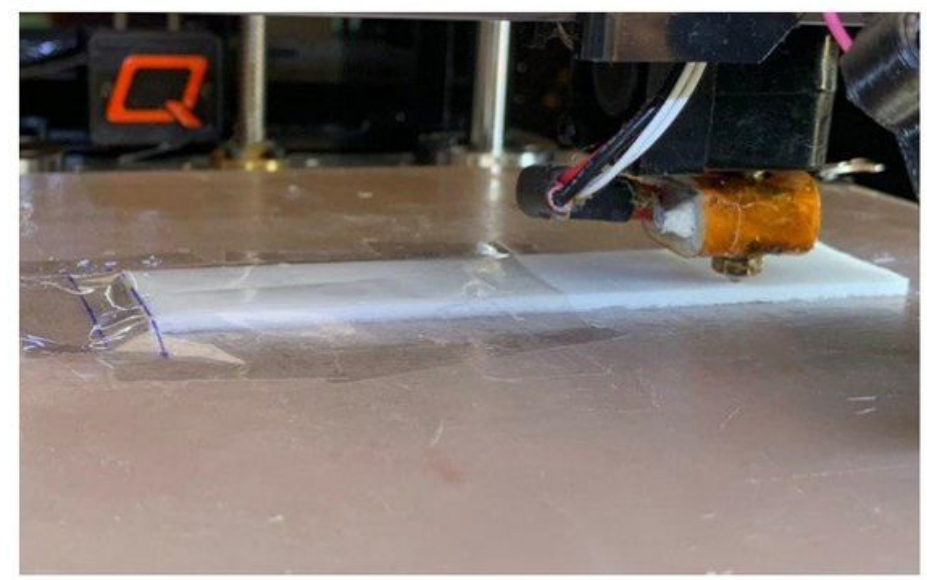

e) Tensile Displacement

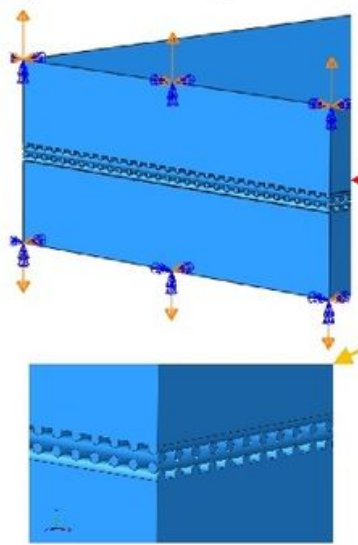

Lattice

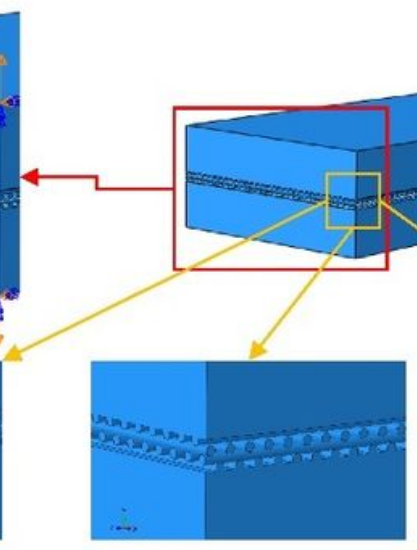

Shifted

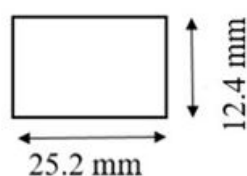

c)
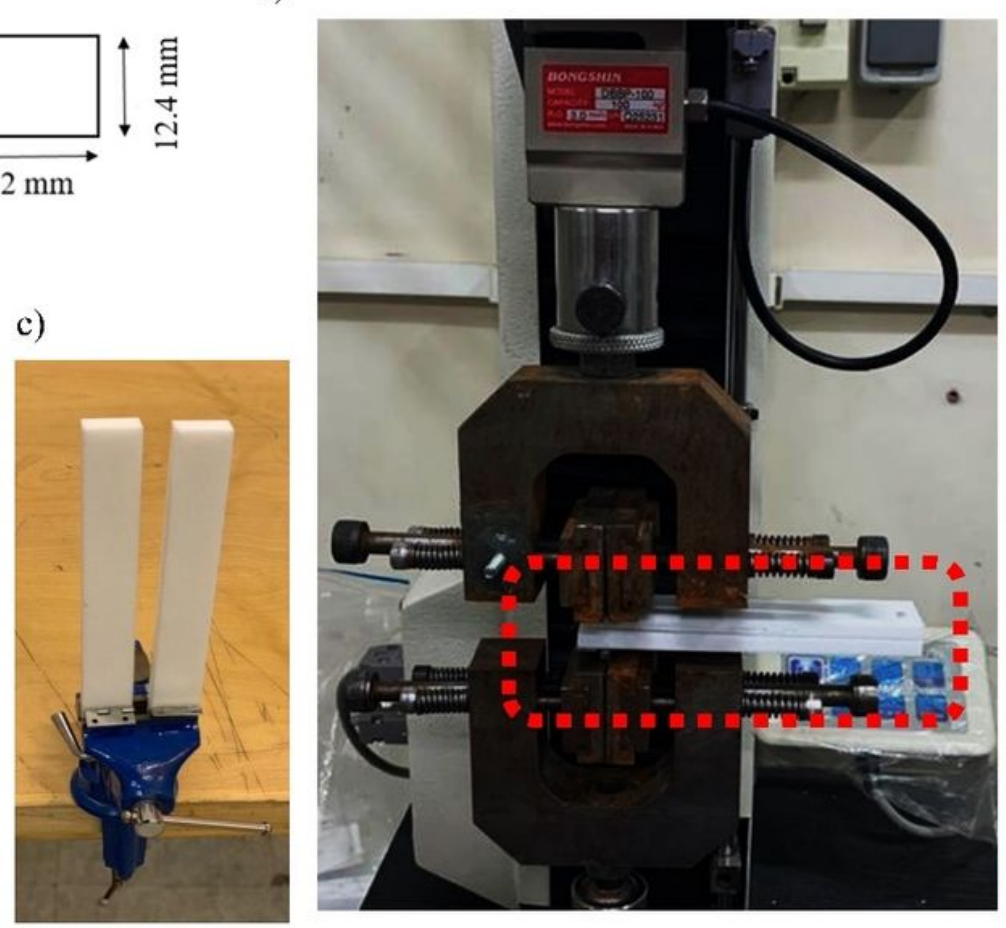

f) ENCASTRE d)

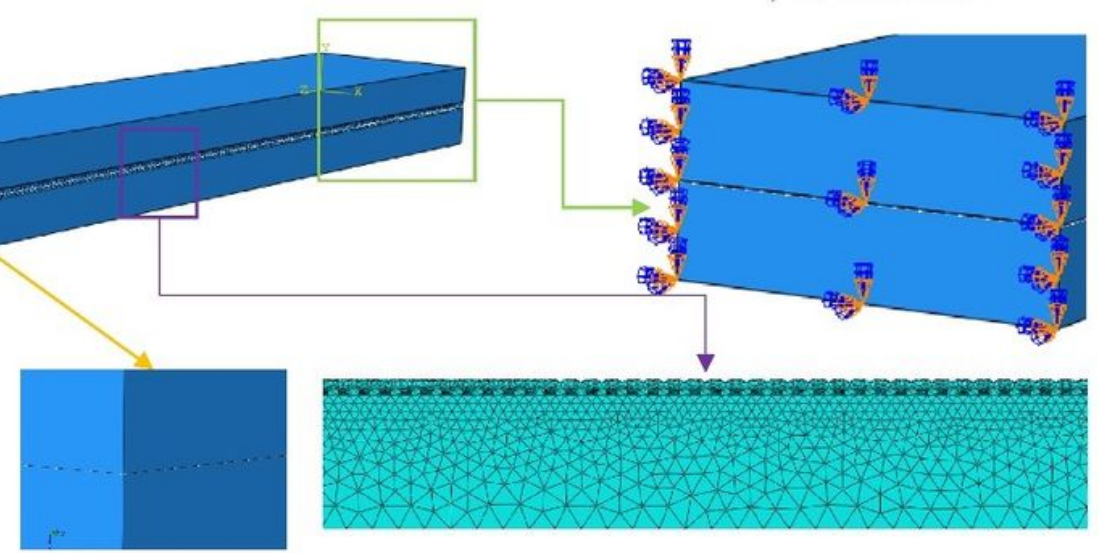

Bulk

\section{Figure 5}

The DCB test specifications in experimental (a-d) and numerical (e-g) studies. (a) The specimen dimensions based on ASTM D5528, (b) Creation of the initial crack during the printing process, (c) Installing piano hinge, (d) Performing DCB test, (e) Tensile displacement to simulate loading regime, (f) Constraining all degree of freedom at the end of DCB specimen, (g) Using quadratic tetrahedral (C3D10) elements for meshing of Lattice, Shifted and Bulk designs. 
a)
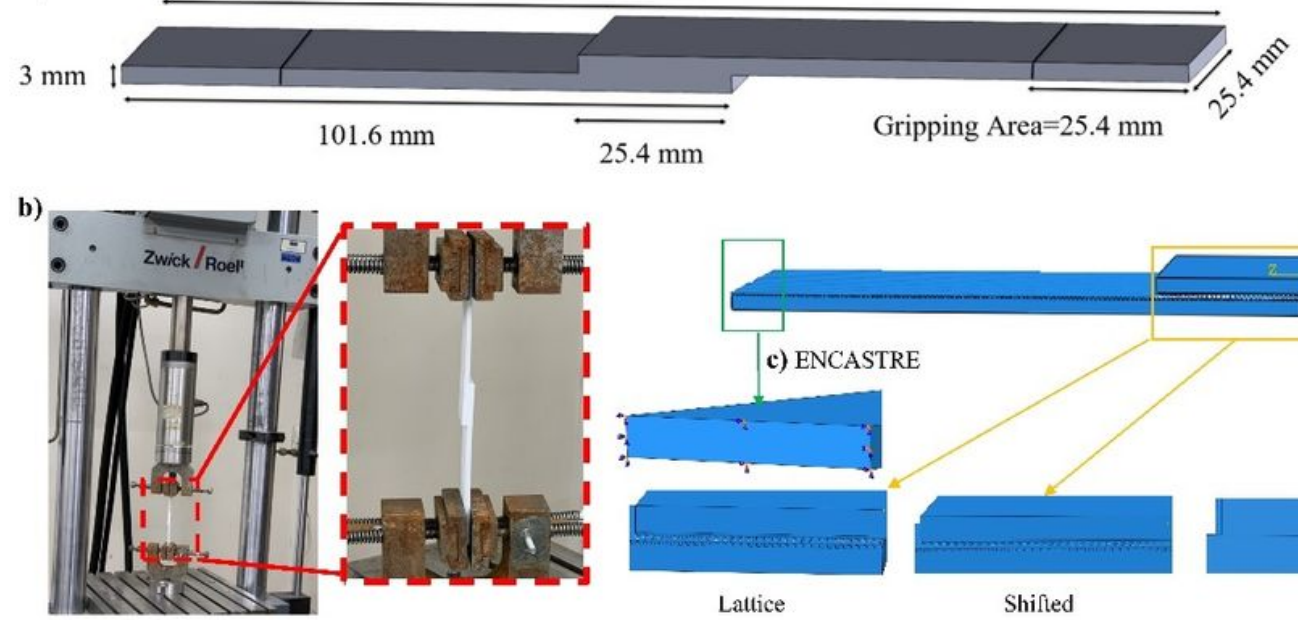

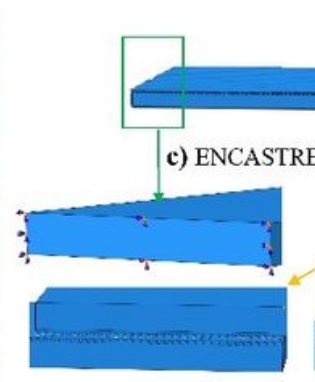

Lattice

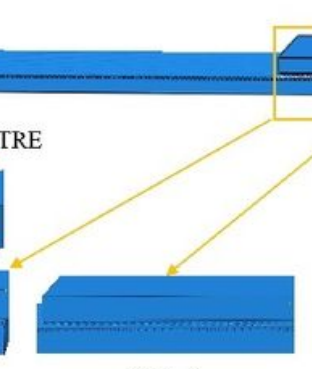

Shifted

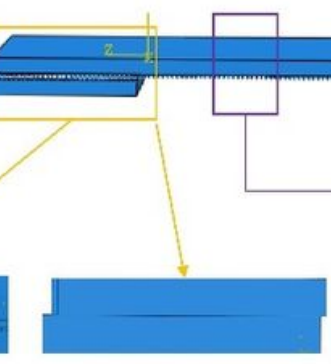

Bulk d) Tensile Displacement

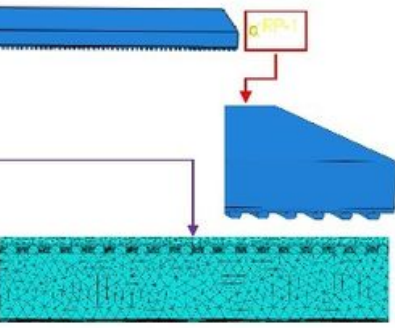

e) 3D Stress Tetrahedral Element

Figure 6

The SLS test specifications in experimental (a \& b) and numerical (c-f) studies. (a) The specimen dimensions based on ASTM D3136, (b) Performing SLS test, (c) Constraining all degree of freedom at the end of the specimen, (d) Tensile displacement to simulate loading regime, (e) Using quadratic tetrahedral (C3D10) elements for meshing of Lattice, Shifted and Bulk designs.

Bulk

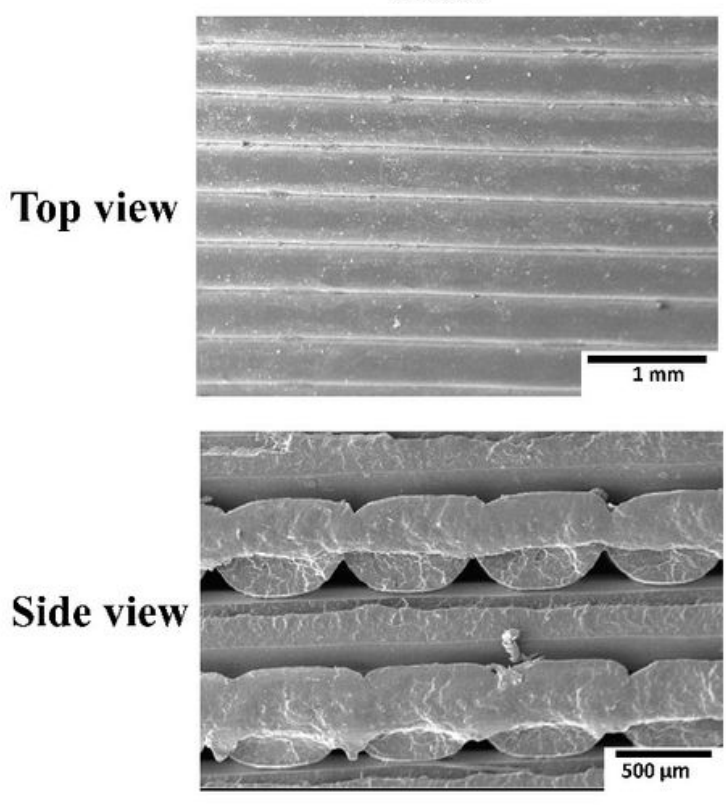

Lattice
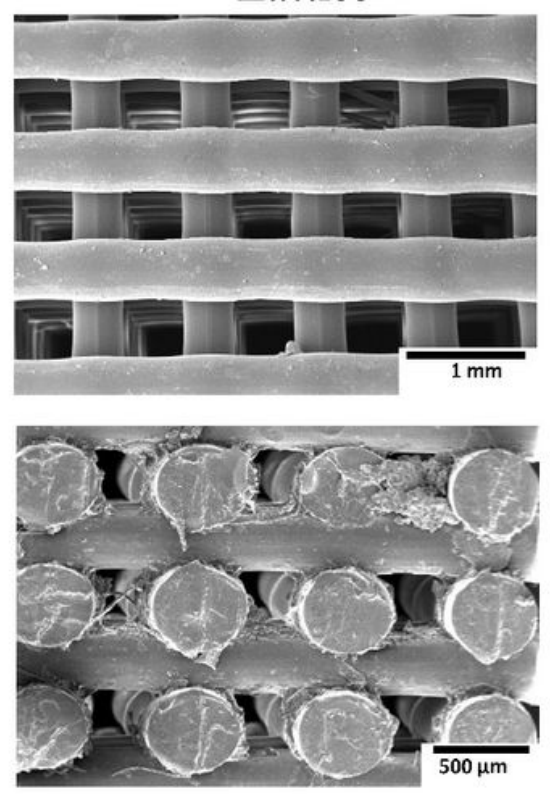

Shifted
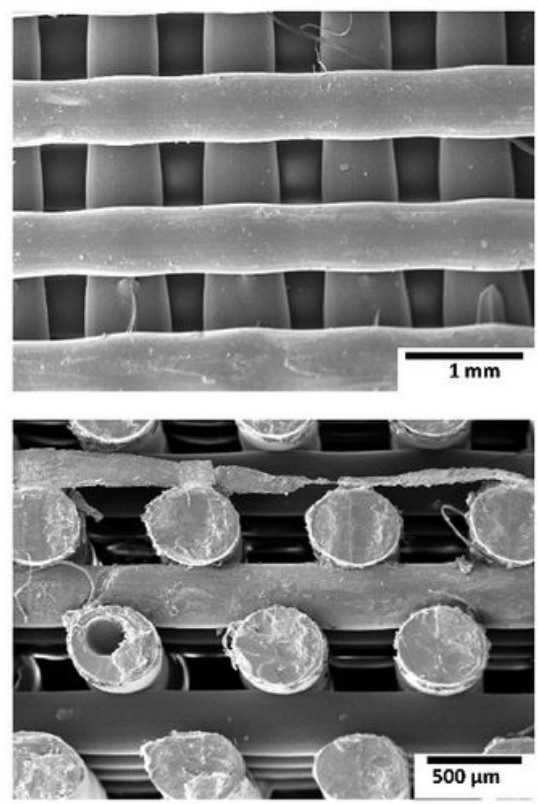

\begin{tabular}{|c|c|}
\hline Layer thickness $(\mathrm{mm})$ & 0.3 \\
\hline Printing speed $(\mathrm{mm} / \mathrm{s})$ & 30 \\
\hline Extrude flow rate & $100 \%$ \\
\hline Bed temperature $\left({ }^{\circ} \mathrm{C}\right)$ & 50 \\
\hline Nozzle temperature $\left({ }^{\circ} \mathrm{C}\right)$ & 195 \\
\hline
\end{tabular}

Design

Lattice

Shifted

Bulk

\section{Strut Diameter $(\mu \mathrm{m}) \quad$ Pore Size $(\mu \mathrm{m}) \quad$ Layer Height $(\mu \mathrm{m})$}

\begin{tabular}{|c|c|c|}
\hline $413.96 \pm 13.58$ & $378.53 \pm 21.40$ & $319.15 \pm 3.46$ \\
\hline $407.65 \pm 15.80$ & $430.17 \pm 24.77$ & $327.68 \pm 6.32$ \\
\hline $409.83 \pm 21.33$ & - & $326.22 \pm 10.24$ \\
\hline
\end{tabular}

Figure 7 
3D printing parameters, SEM images of Bulk, Lattice, and Shifted samples from top and side views, and morphological parameters of strut diameter, pore size, and layer height measured from SEM images.

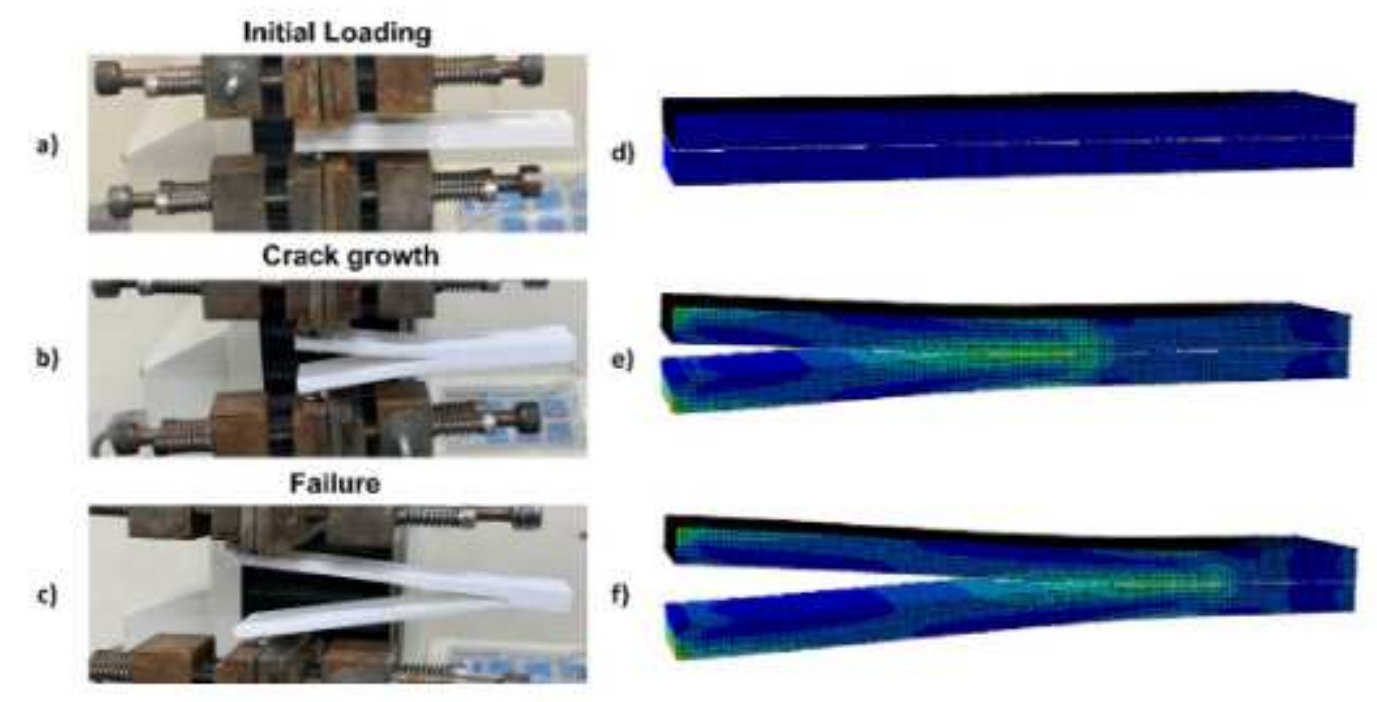

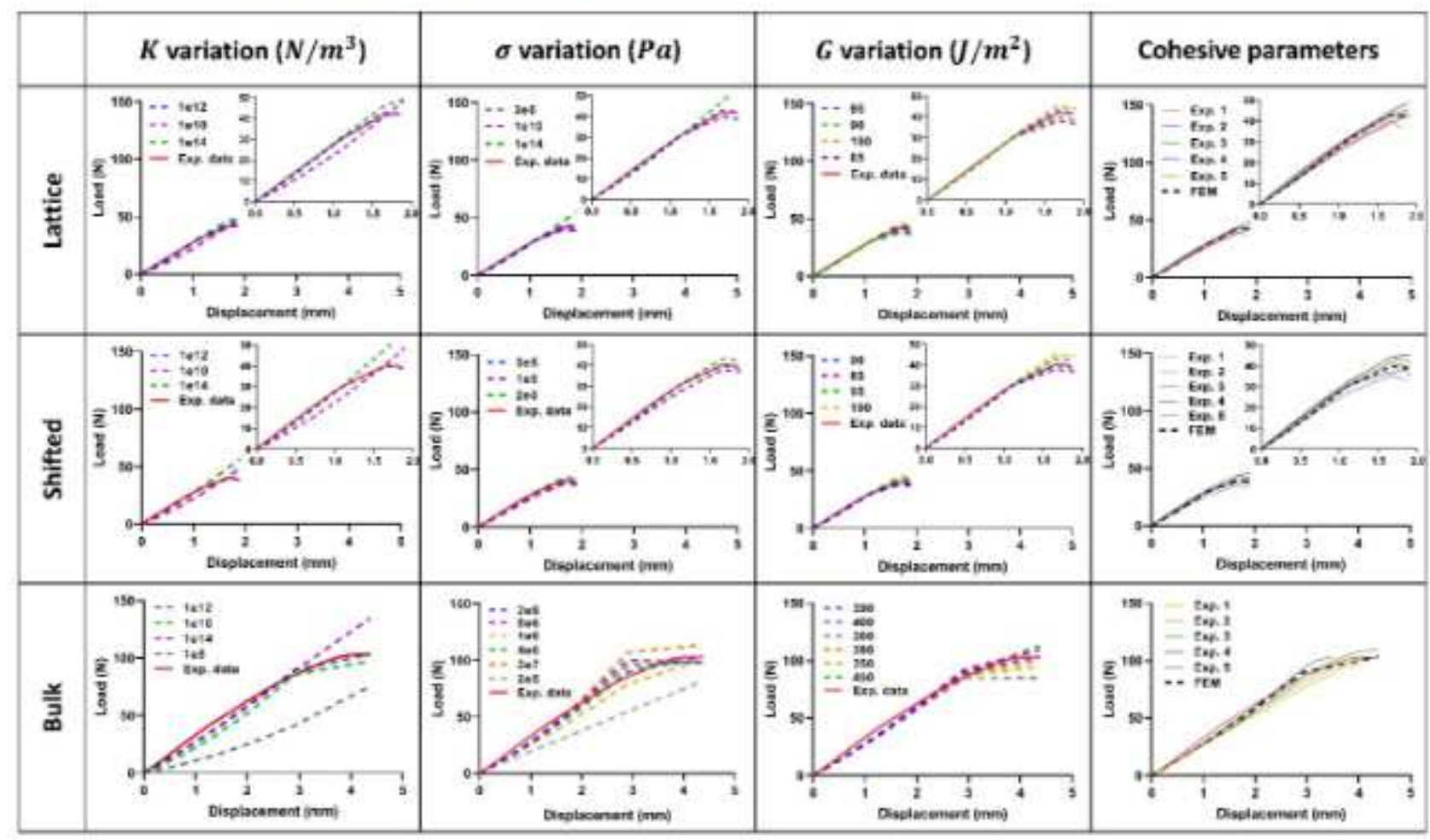

\section{Figure 8}

The double cantilever beam (DCB) test steps and its computational simulation. The load-displacement curves with the variation of cohesive parameters were compared with experimental data, and the best fitting was selected. In the right column, the numerical results with selected cohesive parameters and 5 DCB test results are illustrated. 


\section{Initial Loading}

a)

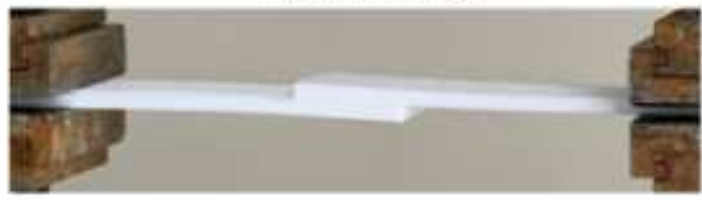

Bending Moment

b)

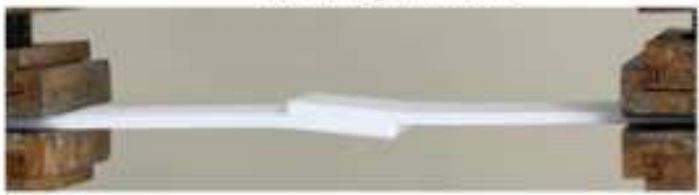

Failure

c)

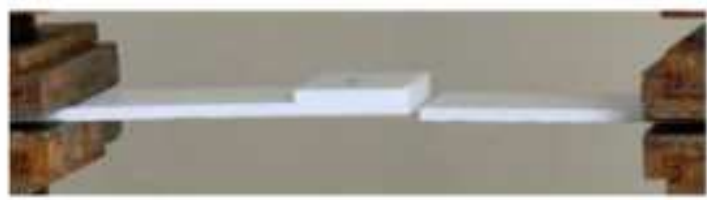

d)

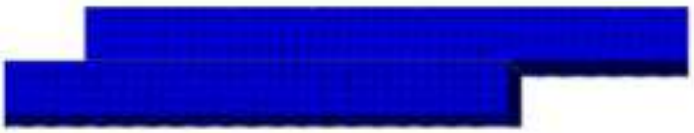

e)

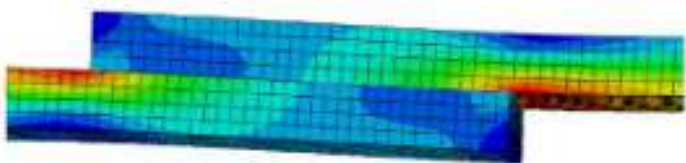

f)

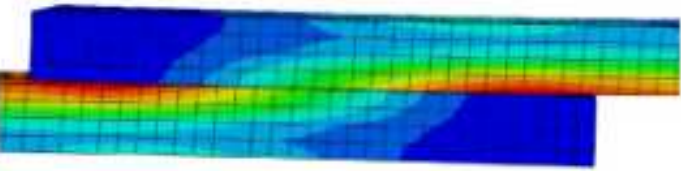

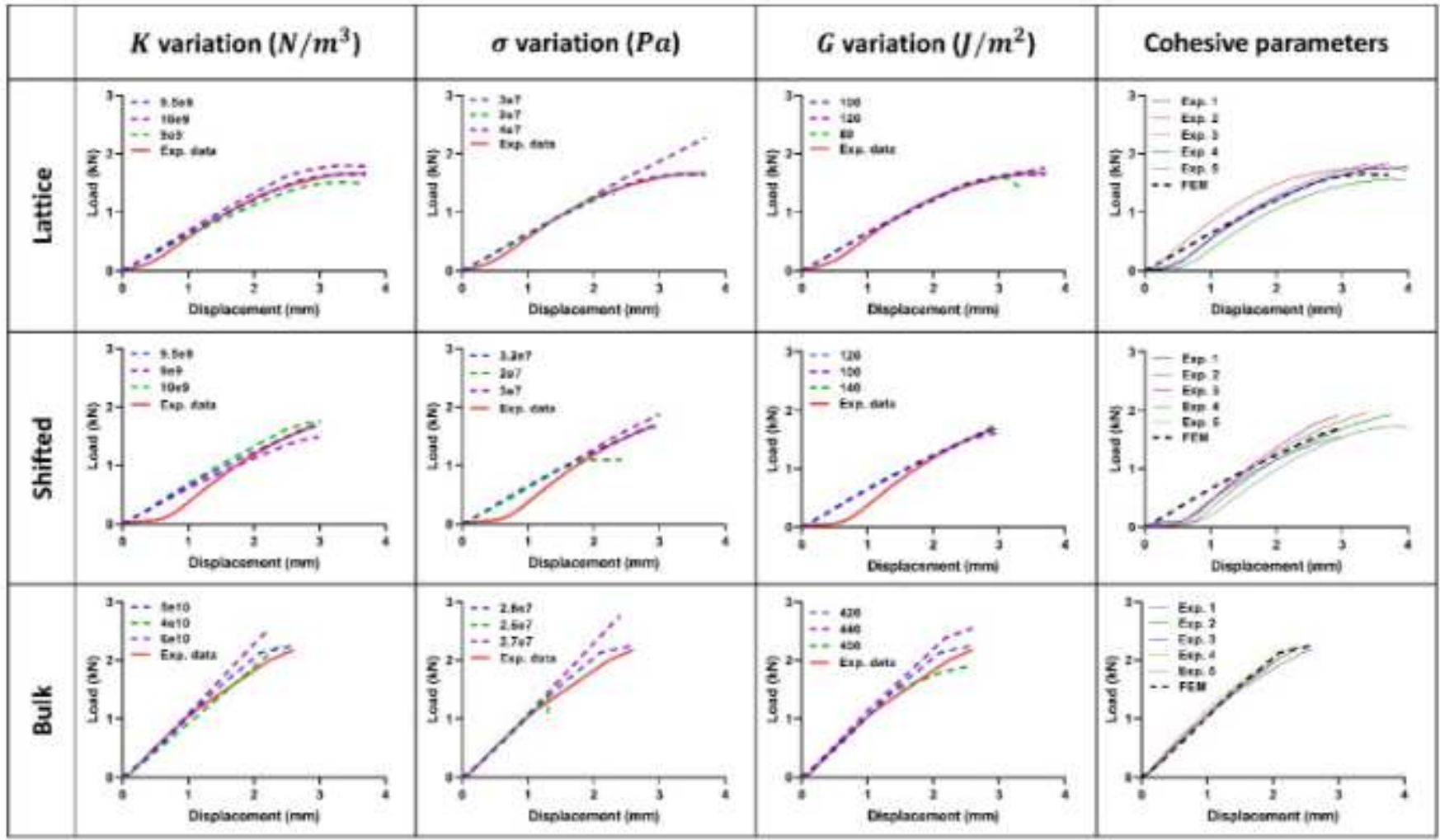

\section{Figure 9}

The single lap shear (SLS) test steps (a-c) and its computational simulation (d-f). The load-displacement curves with the variation of cohesive parameters were compared with experimental data, and the best fitting was selected. In the right column, the numerical results with selected cohesive parameters and 5 SLS test results are illustrated. 


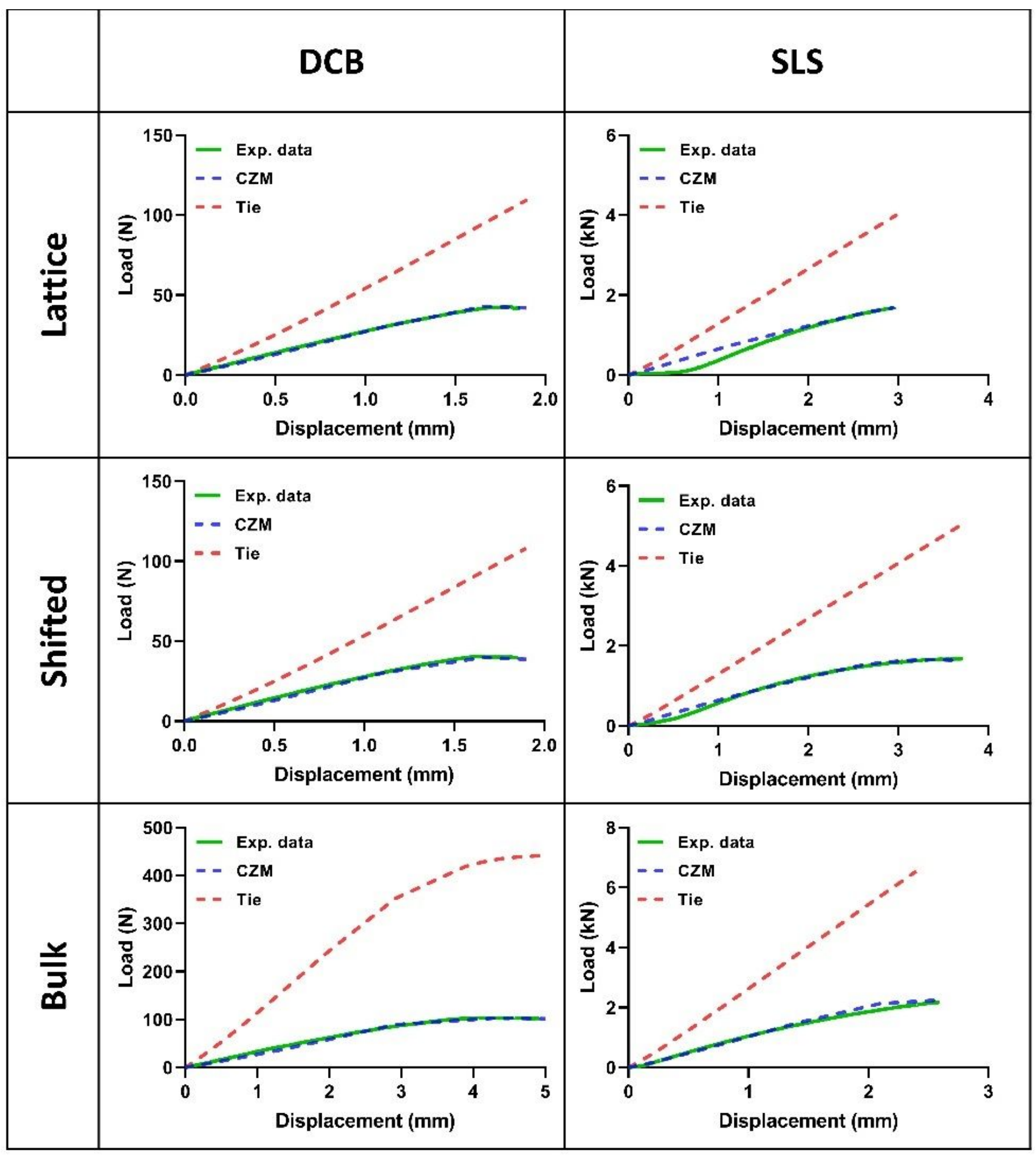

Figure 10

The effect of assuming partially bonded layers by the cohesive zone model (CZM) to simulate interlayer contact for Bulk and porous additive manufacturing parts in comparison to fully bonded (tie constraint in Abaqus software) layers assumption. The modeling of voids and gaps between layers enhanced the computational accuracy using CZM in both Modes I and II. 


\section{Supplementary Files}

This is a list of supplementary files associated with this preprint. Click to download.

- Highlight.docx 